\title{
Complex rotundities and midpoint local uniform rotundity in symmetric spaces of measurable operators
}

\author{
by \\ Mazgorzata Marta Czerwińska and \\ AnNA KamińsKa (Memphis, TN)
}

\begin{abstract}
We investigate the relationships between strongly extreme, complex extreme, and complex locally uniformly rotund points of the unit ball of a symmetric function space or a symmetric sequence space $E$, and of the unit ball of the space $E(\mathcal{M}, \tau)$ of $\tau$-measurable operators associated to a semifinite von Neumann algebra $(\mathcal{M}, \tau)$ or of the unit ball in the unitary matrix space $C_{E}$. We prove that strongly extreme, complex extreme, and complex locally uniformly rotund points $x$ of the unit ball of the symmetric space $E(\mathcal{M}, \tau)$ inherit these properties from their singular value function $\mu(x)$ in the unit ball of $E$ with additional necessary requirements on $x$ in the case of complex extreme points. We also obtain the full converse statements for the von Neumann algebra $\mathcal{M}$ with a faithful, normal, $\sigma$-finite trace $\tau$ as well as for the unitary matrix space $C_{E}$. Consequently, corresponding results on the global properties such as midpoint local uniform rotundity, complex rotundity and complex local uniform rotundity follow.
\end{abstract}

Let $E$ be a symmetric sequence space, and let $C_{E}$ be the unitary matrix space of compact operators acting on Hilbert space, associated with $E$. One of the points of interest in the theory of unitary matrix spaces is to investigate what properties of the symmetric sequence space $E$ are inherited by the unitary matrix space $C_{E}$ [22. It was shown by Arazy in 2] that $E$ is isometrically embedded in $C_{E}$, and that the isometry $V$ can be chosen with respect to any compact operator $x$ in such a way that $V(s(x))=x$, where $s(x)=\left\{s_{n}(x)\right\}_{n=1}^{\infty}$ is the sequence of singular numbers of $x$. Therefore many geometrical properties of $x \in C_{E}$ are also satisfied by $s(x) \in E$. In the same paper Arazy showed that $x \in C_{E}$ is an extreme (resp. smooth, exposed) point of the unit ball in $C_{E}$ if and only if $s(x)$ is an extreme (resp. smooth,

2010 Mathematics Subject Classification: 46B20, 46B28, 47L05, 47L20.

Key words and phrases: symmetric spaces of measurable operators, unitary matrix spaces, strongly extreme points, midpoint local uniform rotundity, complex extreme points, complex rotundity, complex locally uniformly extreme points, complex local uniform rotundity. 
exposed) point of the unit ball in $E$. Lifting the uniform rotundity from $E$ to $C_{E}$ was considered by Tomczak-Jaegermann in [36].

Later on, more general spaces have been considered, namely the symmetric spaces $E(\mathcal{M}, \tau)$ of $\tau$-measurable operators associated to a symmetric Banach function space $E$ and a semifinite von Neumann algebra $(\mathcal{M}, \tau)$. The space $E(\mathcal{M}, \tau)$ consists of all $\tau$-measurable operators $x$ for which the singular value function $\mu(x)$ belongs to $E$, and it is equipped with the norm $\|x\|_{E(\mathcal{M}, \tau)}=\|\mu(x)\|_{E}$. With the development of the theory of symmetric spaces of measurable operators, it was also natural to expect the noncommutative space $E(\mathcal{M}, \tau)$ to reflect the properties of the corresponding symmetric function space $E$. Until now it has been shown that many geometric properties, like rotundity [5], (local) uniform rotundity [6], (uniform) Kadec-Klee property [15, 16], Banach-Saks property [17] and several others lift from $E$ to $E(\mathcal{M}, \tau)$. In particular, Chilin, Krygin and Sukochev [5] characterized the extreme points of the unit ball in $E(\mathcal{M}, \tau)$ in terms of the singular value function $\mu(x)$ in the unit ball of $E$. Later on in [6] they showed that local uniform rotundity and uniform rotundity are inherited by $E(\mathcal{M}, \tau)$ from $E$.

The aim of this paper is to investigate complex extreme points $(\mathbb{C}$-extreme points), complex locally uniformly extreme points ( $\mathbb{C}$-LUR points), strongly extreme points (MLUR points) of the unit ball of $E(\mathcal{M}, \tau)$ and the associated properties of complex rotundity ( $\mathbb{C}$-rotundity), complex local uniform rotundity ( $\mathbb{C}$-LUR), and midpoint local uniform rotundity (MLUR) of $E(\mathcal{M}, \tau)$.

The concepts of $\mathbb{C}$-extreme points and $\mathbb{C}$-rotund spaces have been introduced by Thorp and Whitley in [35] in connection with the strong maximum modulus theorem for vector-valued analytic functions. Its liaison to holomorphic spaces has been further confirmed by Globevnik's work in [21] who investigated complex uniformly rotund spaces and showed among other things that peak points of the ball algebra over a Banach space $X$ are complex extreme points of its unit ball $B_{X}$. Along the same lines are for instance the recent results in [1]. The complex geometric properties also found other applications, for instance in studying the (local) geometry of Banach spaces [10]. Moreover, as has recently been observed [23], $\mathbb{C}$-extreme points and $\mathbb{C}$-rotundity of a complex Banach lattice $E$ are equivalent to upper monotone points and strict monotonicity of its real part $E_{r}$, respectively. That observation can be very useful in studying complex properties in Banach lattices and we will apply it later in this paper.

In 2000, Wang and Teng [37] defined $\mathbb{C}$-LUR points and $\mathbb{C}$-LUR spaces and obtained criteria for this property in the class of Musielak-Orlicz spaces of vector-valued functions. 
The MLUR property in real Banach spaces, its role and relations to other geometric properties are thoroughly discussed in [29].

Observe that complex uniform rotundity in noncommutative spaces has been investigated in [9, 38, where in particular it was shown that the trace class $S_{1}=C_{L_{1}}$ is complex uniformly rotund [9], and that $E(\mathcal{M}, \tau)$ is complex uniformly convexifiable if and only if $E$ has some finite concavity [38].

In Section 1 we provide the terminology and some facts, especially in noncommutative spaces, to be used later.

In Section 2 we prove that if $x$ is order continuous and $E$ is a fully symmetric function space then $x$ is a MLUR point of the unit ball in $E(\mathcal{M}, \tau)$ whenever $\mu(x)$ is a MLUR point of the unit ball in $E$. Furthermore, under the assumption that $E$ is a symmetric function space and the von Neumann algebra $\mathcal{M}$ has a $\sigma$-finite trace we show that if $x$ is a MLUR point then so is $\mu(x)$. Consequently, the MLUR property of a fully symmetric space $E$ lifts to $E(\mathcal{M}, \tau)$, and vice versa, $E$ inherits this property from $E(\mathcal{M}, \tau)$ under the additional assumption that the trace $\tau$ on $\mathcal{M}$ is $\sigma$-finite.

Section 3 is devoted to $\mathbb{C}$-extreme points and $\mathbb{C}$-rotundity. We provide a characterization of $\mathbb{C}$-extreme points of the unit ball in $E(\mathcal{M}, \tau)$, where $E$ is a symmetric function space, analogous to that of extreme points obtained by Chilin, Krygin and Sukochev in [5, 18]. As a consequence we also find that $\mathbb{C}$-rotundity is inherited by $E(\mathcal{M}, \tau)$ from $E$, and we have the opposite implication under the same $\sigma$-finiteness assumption on the trace $\tau$ as for the MLUR property.

In Section 4 we prove analogous results for $\mathbb{C}$-LUR points and the $\mathbb{C}$ LUR property. We show that if $x$ is order continuous and $E$ is a strongly symmetric function space, and if $\mu(x)$ is a $\mathbb{C}$-LUR point of the unit ball in $E$, then $x$ is a $\mathbb{C}$-LUR point of the unit ball in $E(\mathcal{M}, \tau)$. Therefore if $E$ is order continuous then the $\mathbb{C}$-LUR property lifts from $E$ to $E(\mathcal{M}, \tau)$. The opposite implication holds for a symmetric function space $E$ and a von Neumann algebra $\mathcal{M}$ with a $\sigma$-finite trace $\tau$.

Finally in the last section we discuss the definitions of $\mathbb{C}$-MLUR points and $\mathbb{C}$-MLUR spaces that are analogous to those for MLUR points and MLUR spaces in [20] for real Banach spaces. We present several equivalent conditions, and in particular we show that in any complex Banach space these notions are equivalent to $\mathbb{C}$-LUR points and $\mathbb{C}$-LUR spaces, respectively. Therefore $\mathbb{C}$-MLUR and $\mathbb{C}$-LUR are not distinguishable, unlike their real counterparts MLUR and LUR.

1. Preliminaries. As usual, we denote by $\mathbb{C}, \mathbb{R}$, and $\mathbb{N}$ the sets of complex, real, and natural numbers, respectively. Given $z \in \mathbb{C}$, we denote by $\bar{z}$, $\operatorname{Re} z$ and $\operatorname{Im} z$ the conjugate of $z$ and the real and imaginary parts of $z$, respectively. 
Let $I=\mathbb{N}$ or $I=[0, \alpha), 0<\alpha \leq \infty$, be equipped with the counting measure or the Lebesgue measure, respectively. We denote by $L^{0}=L^{0}(I)$ the set of all complex-valued measurable functions on $I$. We say that $\left(E,\|\cdot\|_{E}\right)$ is a Banach lattice if $E=E(I) \subset L^{0}(I)$ is a Banach space with respect to the norm $\|\cdot\|_{E}$ such that whenever $0 \leq f \leq g, f \in L^{0}(I)$ and $g \in E$, then $f \in E$ and $\|f\|_{E} \leq\|g\|_{E}$. When $I=\mathbb{N}, E$ is called a Banach sequence lattice, and when $I=[0, \alpha)$, it is often called a Banach function lattice.

For a Banach lattice $E$ over the field of complex numbers we define its real part as

$$
E_{r}=\{f \in E: \operatorname{Im}(f)=0\},
$$

with the norm induced from $E$. Denote by $E^{+}$the cone of positive elements in $E$, and by $S_{E^{+}}$and $B_{E^{+}}$the unit sphere and the unit ball of positive elements of $E$, respectively. Recall that a Banach lattice $E$ satisfies the Fatou property whenever for any $f_{n} \in E$ and $f \in L^{0}$, if $f_{n} \uparrow f$ a.e. and $\sup _{n}\left\|f_{n}\right\|_{E}<\infty$ then $f \in E$ and $\left\|f_{n}\right\|_{E} \uparrow\|f\|_{E}$. An element $f \in E$ is called order continuous if for every $0 \leq f_{n} \leq|f|$ such that $f_{n} \downarrow 0$ a.e. we have $\left\|f_{n}\right\|_{E} \downarrow 0$. We say that $E$ is order continuous if every element in $E$ is order continuous.

Given $f \in L^{0}[0, \alpha), 0<\alpha \leq \infty$, the distribution function $d_{f}$ of the function $f$ is given by

$$
d_{f}(\lambda)=m\{t>0:|f(t)|>\lambda\}, \quad \lambda \geq 0,
$$

and its decreasing rearrangement $\mu_{t}(f)$ is defined as

$$
\mu_{t}(f)=\inf \left\{s>0: d_{f}(s) \leq t\right\}, \quad t \geq 0 .
$$

We denote by $\mu(f)$ the function $t \mapsto \mu_{t}(f), t \geq 0$, and set $\mu_{\infty}(f)=$ $\lim _{t \rightarrow \infty} \mu_{t}(f)$. The symbol $E_{0}$ will stand for the space of all elements $f$ of $E$ for which $\mu_{\infty}(f)=0$.

We say that $\left(E,\|\cdot\|_{E}\right)$ is a symmetric (function) space on $[0, \alpha)$ if $E$ is a Banach function lattice on $[0, \alpha)$ and $\|f\|_{E}=\|\mu(f)\|_{E}$ for all $f \in E$. Analogously, when $E$ is a Banach sequence lattice, $E$ is called a symmetric (sequence) space whenever $\|\mu(x)\|_{E}=\|x\|_{E}$, where $\mu(x)$ is the decreasing rearrangement of the sequence $x \in E$.

We say that $f$ is submajorized by $g$, and write $f \prec g$, whenever

$$
\int_{0}^{s} \mu_{t}(f) d t \leq \int_{0}^{s} \mu_{t}(g) d t \quad \text { for every } s>0 .
$$

A symmetric space $E$ is strongly symmetric if for $f, g \in E$, if $f \prec g$ then $\|f\|_{E} \leq\|g\|_{E}$. If a symmetric space $E$ satisfies the Fatou property or $E$ is order continuous, then $E$ is fully symmetric, that is, if $f \in L^{0}, g \in E$ and $f \prec g$ then $f \in E$ and $\|f\|_{E} \leq\|g\|_{E}$. For this and all other facts and definitions concerning Banach lattices we refer to monographs [3, 18, 27]. 
Let $H$ be a complex Hilbert space and $B(H)$ the space of bounded linear operators from $H$ to $H$. Moreover, we denote by $L(H)$ the space of all linear operators $x: \mathcal{D}(x) \rightarrow H$, where $\mathcal{D}(x) \subset H$ is the domain of $x$. Let $\mathcal{M} \subset B(H)$ be a semifinite von Neumann algebra with a faithful, normal, semifinite trace $\tau$, and let $\mathcal{P}(\mathcal{M})$ be the lattice of all projections in $\mathcal{M}$. If $x \in \mathcal{M}$ then $\|x\|_{\mathcal{M}}$ denotes the operator norm in $B(H)$. A nonzero projection $p \in \mathcal{P}(\mathcal{M})$ is called minimal if $q \in \mathcal{P}(\mathcal{M})$ and $q \leq p$ imply that $q=p$ or $q=0$. The projections $p$ and $q$ are said to be equivalent (relative to the von Neumann algebra $\mathcal{M}$ ), denoted by $p \sim q$, if there exists a partial isometry $v \in \mathcal{M}$ such that $p=v^{*} v$ and $q=v v^{*}$. The von Neumann algebra $\mathcal{M}$ is called nonatomic if $\mathcal{M}$ contains no minimal projections [34]. A densely defined closed operator $x$, affiliated with $\mathcal{M}$, is called $\tau$-measurable if for each $\epsilon>0$ there exists $p \in \mathcal{P}(\mathcal{M})$ such that $p(H) \subset \mathcal{D}(x)$ and $\tau\left(p^{\perp}\right)<\epsilon$, where $p^{\perp}=\mathbf{1}-p$ and $\mathbf{1}$ is the unit element in $\mathcal{M}$. The set $S(\mathcal{M}, \tau)$ of all $\tau$-measurable operators is a $*$-algebra with respect to the strong sum, the strong product, and the adjoint operator. It is well known that in the commutative case the space $S([0, \alpha), m)$ of measurable operators may be identified with

$$
\left\{f \in L^{0}[0, \alpha): m([0, \alpha) \backslash A)<\infty \text { and } f \chi_{A} \in L_{\infty}[0, \alpha) \text { for some } A\right\},
$$

where $\alpha=\tau(\mathbf{1})$. For $x \in S(\mathcal{M}, \tau)$, we denote by $n(x)$ the null projection onto $\operatorname{Ker} x$. Then $s(x)=\mathbf{1}-n(x)$ is called the support projection, that is, a projection onto $\mathrm{Ker}^{\perp} x$. Furthermore, if $x=u|x|$ is a polar decomposition of $x$, then $s(x)=u^{*} u$ and $s\left(x^{*}\right)=u u^{*}$. For every subset $X \subset S(\mathcal{M}, \tau)$ we shall denote the set of all self-adjoint (respectively, positive) elements of $X$ by $X_{h}$ (respectively, $X^{+}$). By a positive operator $x$ we mean a selfadjoint operator such that $\langle x \xi, \xi\rangle \geq 0$ for all $\xi \in \mathcal{D}(x)$. Hence $S_{h}(\mathcal{M}, \tau)$ is equipped with the partial order $\geq$, generated by the proper cone $S^{+}(\mathcal{M}, \tau)$. If $\left\{x_{n}\right\} \subset S_{h}(\mathcal{M}, \tau)$ is decreasing and $x=\inf _{n} x_{n}$ exists, then we write $x_{n} \downarrow_{n} x$. For an operator $x \in S(\mathcal{M}, \tau)$, the function $\mu_{t}(x)$ defined by

$$
\mu_{t}(x)=\inf \left\{s \geq 0: \tau\left(e^{|x|}(s, \infty)\right) \leq t\right\}, \quad t \geq 0,
$$

where $e^{|x|}(s, \infty)$ is the spectral projection of $|x|=\left(x^{*} x\right)^{1 / 2}$ corresponding to the interval $(s, \infty)$, is called the decreasing rearrangement of $x$ or the generalized singular value function of $x$. The function $\tau\left(e^{|x|}(s, \infty)\right)$ is called the spectral distribution of $x$. We shall use the notation $\mu(x)$ for the function $\mu_{t}(x), t \in \mathbb{R}_{+}$. Observe that in the commutative case, measurable operators are identified with some measurable functions $f$, and then $\mu(f)$ is the usual decreasing rearrangement of $f$. Notice that $\mu_{t}(x)=0$ for $t \in[\alpha, \infty)$, if $\alpha<\infty$. Put $\mu_{\infty}(x)=\lim _{t \rightarrow \infty} \mu_{t}(x)$ for $x \in S(\mathcal{M}, \tau)$. Then the set

$$
S_{0}(\mathcal{M}, \tau)=\left\{x \in S(\mathcal{M}, \tau): \mu_{\infty}(x)=0\right\}
$$

is a $*$-subalgebra in $S(\mathcal{M}, \tau)$. 
Let $x, y \in S(\mathcal{M}, \tau)$. We say that $x$ is submajorized by $y$, and write $x \prec y$, whenever $\mu(x) \prec \mu(y)$. It is well known that $\mu(x+y) \prec \mu(x)+\mu(y)$ and $\mu(x)-\mu(y) \prec \mu(x-y)$, for all $x, y \in S(\mathcal{M}, \tau)$.

Recall the following properties of the singular value function. Although the first two properties are certainly well known we sketch their proofs for the sake of completeness.

Proposition 1.1.

(1) For $x \in S(\mathcal{M}, \tau), \mu\left(|x|+\mu_{\infty}(x) n(x)\right)=\mu(x)$.

(2) If $x \in S(\mathcal{M}, \tau)$ and $|x| \geq \mu_{\infty}(x) s(x)$ then $\mu\left(|x|-\mu_{\infty}(x) s(x)\right)=$ $\mu(x)-\mu_{\infty}(x)$.

(3) [5, Proposition 2.2] If $x, y \in S^{+}(\mathcal{M}, \tau), y \neq 0$ and $x \geq \mu_{\infty}(x) \mathbf{1}$, then there exists $s>0$ such that $\mu_{s}(x)<\mu_{s}(x+y)$.

(4) [33, Proposition 3] If $x, y \in S(\mathcal{M}, \tau), y=y^{*}, x \geq 0$, then $\mu_{t}(x) \leq$ $\mu_{t}(x+i y)$ for all $t>0$.

(5) [5, Proposition 3.5] If $x, y \in S(\mathcal{M}, \tau), y=y^{*}, x \geq \mu_{\infty}(x) 1$ and $\mu(x+i y)=\mu(x)$, then $y=0$.

Proof. (1) For $x \in S(\mathcal{M}, \tau)$, consider the real-valued function $f(t)=$ $t+\mu_{\infty}(x) \chi_{\{0\}}(t), t \geq 0$. Then, by functional calculus, $f(|x|)=|x|+\mu_{\infty}(x) n(x)$ $\geq 0$ and for $\lambda>0$,

$e^{|x|+\mu_{\infty}(x) n(x)}(\lambda, \infty)=e^{|x|}\left(f^{-1}(\lambda, \infty)\right)= \begin{cases}e^{|x|}(\lambda, \infty) & \text { if } \lambda \geq \mu_{\infty}(x), \\ e^{|x|}((\lambda, \infty) \cup\{0\}) & \text { if } \lambda<\mu_{\infty}(x) .\end{cases}$

Since $\mu_{\infty}(x)=\inf \left\{\lambda: \tau\left(e^{|x|}(\lambda, \infty)\right)<\infty\right\}$, we have $\tau\left(e^{|x|}(\lambda, \infty)\right)=\infty$ for all $\lambda<\mu_{\infty}(x)$. Hence also $\tau\left(e^{|x|+\mu_{\infty}(x) n(x)}(\lambda, \infty)\right)=\infty$ for $\lambda<\mu_{\infty}(x)$, and so $\mu_{t}\left(|x|+\mu_{\infty}(x) n(x)\right)=\mu_{t}(x)$ for $t>0$.

(2) Let $x \in S(\mathcal{M}, \tau)$ and $|x| \geq \mu_{\infty}(x) s(x)$. Consider the function $f(t)=$ $t-\mu_{\infty}(x) \chi_{(0, \infty)}$. Then $f(|x|)=|x|-\mu_{\infty}(x) s(x) \geq 0$ and for all $\lambda>0$, $e^{|x|-\mu_{\infty}(x) s(x)}(\lambda, \infty)=e^{f(|x|)}(\lambda, \infty)=e^{|x|}\left(f^{-1}(\lambda, \infty)\right)=e^{|x|}\left(\lambda+\mu_{\infty}(x), \infty\right)$. Thus $\left\||x|-\mu_{\infty}(x) s(x)\right\|=|x|-\mu_{\infty}(x) s(x)$, and so

$$
\begin{aligned}
\mu_{t}\left(|x|-\mu_{\infty}(x) s(x)\right) & =\inf \left\{\lambda \geq 0: \tau\left(e^{|x|-\mu_{\infty}(x) s(x)}(\lambda, \infty)\right) \leq t\right\} \\
& =\inf \left\{\lambda \geq 0: \tau\left(e^{|x|}\left(\lambda+\mu_{\infty}(x), \infty\right)\right) \leq t\right\}, \quad t>0 .
\end{aligned}
$$

Therefore for all $t>0, \mu_{t}\left(|x|-\mu_{\infty}(x) s(x)\right)=\mu_{t}(x)-\mu_{\infty}(x)$.

Let $\left(E,\|\cdot\|_{E}\right)$ be a symmetric space on $[0, \alpha), \alpha=\tau(\mathbf{1})$. Denote by $E(\mathcal{M}, \tau)$ the set of all $x \in S(\mathcal{M}, \tau)$ such that $\mu(x) \in E$. It has recently been shown [25] in full generality that $\|\mu(x)\|_{E}$ defines a norm on the symmetric space $E(M, \tau)$ of measurable operators. In addition, if $\left(E,\|\cdot\|_{E}\right)$ is a Banach space then $E(M, \tau)$ equipped with the norm $\|x\|_{E(\mathcal{M}, \tau)}=\|\mu(x)\|_{E}$ is also a Banach space. 
The measure topology in $S(\mathcal{M}, \tau)$ is determined by a fundamental system $\{U(\epsilon, \delta)\}$ of neighborhoods of zero, where

$$
U(\epsilon, \delta)=\left\{x \in S(\mathcal{M}, \tau):\|x p\|_{\mathcal{M}} \leq \epsilon, \tau\left(p^{\perp}\right) \leq \delta \text { for some } p \in \mathcal{P}(\mathcal{M})\right\} .
$$

The notation $x_{n} \stackrel{\tau}{\rightarrow} x$ will be used to indicate the convergence of the sequence $x_{n}$ to $x$ in the measure topology generated by the trace $\tau$. It is well known [19. Lemma 3.4] that $x_{n} \stackrel{\tau}{\rightarrow} x$ if and only if $\mu_{t}\left(x_{n}-x\right) \rightarrow 0$ a.e.

A linear subspace $X$ in $S(\mathcal{M}, \tau)$ provided with a Banach norm $\|\cdot\|_{X}$ is called a symmetric space on $(\mathcal{M}, \tau)$ if it follows from $x \in X, y \in S(\mathcal{M}, \tau)$, and $\mu_{t}(y) \leq \mu_{t}(x)$ for every $t>0$ that $y \in X$ and $\|y\|_{X} \leq\|x\|_{X}$. A symmetric space $X$ is called strongly symmetric if for $x, y \in X, y \prec x$ implies $\|y\|_{X} \leq\|x\|_{X}$. If from $y \in S(\mathcal{M}, \tau), x \in X$ and $y \prec x$ it follows that $y \in X$ and $\|y\|_{X} \leq\|x\|_{X}$ then $X$ is called fully symmetric.

An operator $x \in E(\mathcal{M}, \tau)$ is said to be order continuous in $E(\mathcal{M}, \tau)$ if for all sequences $\left\{x_{n}\right\} \subset S(\mathcal{M}, \tau)$, whenever $|x| \geq x_{n} \downarrow 0$ then $\left\|x_{n}\right\|_{E(\mathcal{M}, \tau)} \rightarrow 0$. We will denote by $E(\mathcal{M}, \tau)^{\text {oc }}$ the set of all order continuous elements in $E(\mathcal{M}, \tau)$. If $E(\mathcal{M}, \tau)^{\mathrm{oc}}=E(\mathcal{M}, \tau)$ then we say that $E(\mathcal{M}, \tau)$ is an order continuous space. It is worth mentioning that $E(\mathcal{M}, \tau)^{\mathrm{oc}}$ is a $*$-ideal of $E(\mathcal{M}, \tau)$ which is norm closed and is contained in $S_{0}(\mathcal{M}, \tau)$. Furthermore, if $x$ is order continuous then $\left\|x p_{n}\right\|_{E(\mathcal{M}, \tau)} \rightarrow 0$ for every sequence $\left\{p_{n}\right\} \subset \mathcal{P}(\mathcal{M})$ satisfying $p_{n} \downarrow_{n} 0$. For more details we refer the reader to [18].

It is well known that if $E$ is a strongly [fully] symmetric space, then $E(\mathcal{M}, \tau)$ is a strongly [fully] symmetric space on $(\mathcal{M}, \tau)$. Moreover, if $E$ satisfies the Fatou property (or is order continuous), then so is $E(\mathcal{M}, \tau)$. In particular, if $E$ is a symmetric space on $[0, \alpha)$ which is order continuous or satisfies the Fatou property, then it is fully symmetric, and therefore $E(\mathcal{M}, \tau)$ is fully symmetric. Also note that every order continuous symmetric space on $(\mathcal{M}, \tau)$ is embedded in $S_{0}(\mathcal{M}, \tau)$ [7]. If $\mathcal{M}$ is a nonatomic von Neumann algebra then for any symmetric space $E(\mathcal{M}, \tau)$ we have $L_{1}(\mathcal{M}, \tau) \cap \mathcal{M} \subset$ $E(\mathcal{M}, \tau) \subset L_{1}(\mathcal{M}, \tau)+\mathcal{M}$.

For the theory of operator algebras we refer to [24, 34, for noncommutative Banach function spaces to [30, 18, 13, 12, 11], and for unitary matrix spaces to [22].

Let $E \subset c_{0}$ be a symmetric sequence space, and $C_{E}$ be the unitary matrix space of compact operators on the Hilbert space $H$ associated with $E$. Recall that $C_{E}$ is a subspace of the Banach space of compact operators $K(H) \subset$ $B(H)$ for which the sequence $s(x)=\left\{s_{n}(x)\right\}_{n=1}^{\infty}$ of singular numbers is in $E$, and it is equipped with the norm $\|x\|_{C_{E}}=\|s(x)\|_{E}$. Let $G$ be the set of all real functions $f \in L^{1}(0, \infty)+L^{\infty}(0, \infty)$ such that

$$
\pi(f)=\left\{\int_{n-1}^{n} \mu_{t}(f) d t\right\}_{n=1}^{\infty} \in E,
$$


and set $\|f\|_{G}=\|\pi(f)\|_{E}$. If $E$ is order continuous then $\left(G,\|\cdot\|_{G}\right)$ is an order continuous symmetric function space on $[0, \infty)$ [7, Proposition 6.1]. It is well known that $S(B(H), \operatorname{tr})=B(H)$, where $\operatorname{tr}$ is the canonical trace on $B(H)$, and the convergence $x_{n} \stackrel{\operatorname{tr}}{\longrightarrow} x$ is equivalent to the norm convergence $\left\|x-x_{n}\right\|_{B(H)} \rightarrow 0$, for $x, x_{n} \in B(H)$. Since $E \subset c_{0}$, we have $E \neq \ell^{\infty}$. Then the symmetric space $G(B(H)$, tr) of measurable operators is a proper two-sided *-ideal in $B(H)$ and therefore it is contained in $K(H)$. Thus for any $x \in G(B(H), \operatorname{tr})$ the singular value function $\mu(x)$ is of the form $\mu_{t}(x)=$ $\sum_{n=1}^{\infty} s_{n}(x) \chi_{[n-1, n)}(t), t \geq 0$. Therefore the spaces $C_{E}$ and $G(B(H), \operatorname{tr})$ coincide as sets and they have identical norms.

Given any linear operator $x: H \rightarrow H$ and a subspace $H_{0} \subset H$, we denote by $\left.x\right|_{H_{0}}$ the restriction of $x$ to $H_{0}$. For a von Neumann algebra $\mathcal{M} \subset B(H)$ and a projection $p \in \mathcal{P}(\mathcal{M})$ define

$$
\mathcal{M}_{p}=\left\{x_{p}: x \in \mathcal{M}\right\},
$$

where $x_{p}=\left.p x\right|_{p(H)}$. It is well known that $\mathcal{M}_{p}$ is a von Neumann algebra contained in $B(p(H))$, with the unit element $p$. We define $\tau_{p}: \mathcal{M}_{p}^{+} \rightarrow[0, \infty]$ by setting

$$
\tau_{p}\left(x_{p}\right)=\tau(p x p),
$$

where $x \in \mathcal{M}^{+}$. Thus for any $y \in \mathcal{M}_{p}^{+}, \tau_{p}(y)=\tau_{p}\left(x_{p}\right)=\tau(p x p)$, where $y=\left.p x\right|_{p(H)}=x_{p}$ for some $x \in \mathcal{M}^{+}$. It is well known that $\tau_{p}$ is a semifinite, normal, faithful trace on $\mathcal{M}_{p}^{+}$, and it is finite if and only if $\tau(p)<\infty$. Furthermore, if $\mathcal{M}$ is nonatomic, then $\mathcal{M}_{p}$ is also nonatomic. Let

$$
p S(\mathcal{M}, \tau) p=\{p x p: x \in S(\mathcal{M}, \tau)\} .
$$

Then $p S(\mathcal{M}, \tau) p$ is a $*$-subalgebra of $S(\mathcal{M}, \tau)$ with the unit element $p$. It is also well known [18, Chapter 3, Section 7] that if $x \in S(\mathcal{M}, \tau)$ then $x_{p} \in S\left(\mathcal{M}_{p}, \tau_{p}\right)$, and the map $\Phi_{p}: x \mapsto x_{p}, x \in p S(\mathcal{M}, \tau) p$, is a unital *isomorphism from $p S(\mathcal{M}, \tau) p$ onto $S\left(\mathcal{M}_{p}, \tau_{p}\right)$. Moreover for any $x \in S(\mathcal{M}, \tau)$ we have $e^{\left|x_{p}\right|}(s, \infty)=e^{|p x p|}(s, \infty), s \geq 0$, and consequently $\mu^{\tau_{p}}\left(x_{p}\right)=$ $\mu(p x p)$, where $\mu^{\tau_{p}}\left(x_{p}\right)$ is the singular value function of $x_{p}$ computed with respect to the reduced von Neumann algebra $\mathcal{M}_{p}$ and the trace $\tau_{p}$.

The following two results will be very useful to show that certain properties of $x$ in $E(\mathcal{M}, \tau)$ are inherited by $\mu(x)$.

Proposition $1.2([18,13])$. Suppose that $\mathcal{M}$ is a nonatomic von Neumann algebra with a faithful, normal, $\sigma$-finite trace $\tau$. Let $x \in L_{1}(\mathcal{M}, \tau)+\mathcal{M}$ and $x \in S_{0}^{+}(\mathcal{M}, \tau)$. Then there exist a nonatomic commutative von Neumann subalgebra $\mathcal{N} \subset \mathcal{M} \subset B(H)$ and a $*$-isomorphism $V$ from the $*$ algebra $S([0, \tau(\mathbf{1})), m)$ into the $*$-algebra $S(\mathcal{N}, \tau)$ such that

$$
V(\mu(x))=x \quad \text { and } \quad \mu(V(f))=\mu(f) \quad \text { for all } f \in S([0, \tau(\mathbf{1})), m) .
$$


Consequently, the Banach function lattice $E=E[0, \tau(\mathbf{1}))$ is isometrically embedded into $E(\mathcal{M}, \tau)$.

Proposition 1.3. Suppose that $\mathcal{M}$ is a nonatomic von Neumann algebra with a faithful, normal, $\sigma$-finite trace $\tau$. Let $x \in S_{0}^{+}(\mathcal{M}, \tau)$ and $p \in$ $\mathcal{P}(\mathcal{M})$ be such that $\tau(p)=\infty$ and $x p=p x=x$. Then there exists a $*$-isomorphism $W$ from the *-algebra $S([0, \infty), m)$ into the $*$-algebra $p S(\mathcal{M}, \tau) p$ such that

$$
W(\mu(x))=x \quad \text { and } \quad \mu(W(f))=\mu(f) \quad \text { for all } f \in S([0, \infty), m) .
$$

Proof. Let $x \in S_{0}^{+}(\mathcal{M}, \tau)$ and $x p=p x=x$. Then $x_{p} \in S_{0}^{+}\left(\mathcal{M}_{p}, \tau_{p}\right)$. In fact by the remarks before, $x_{p} \in S^{+}\left(\mathcal{M}_{p}, \tau_{p}\right)$ and $\mu^{\tau_{p}}\left(x_{p}\right)=\mu(p x p)=\mu(x)$. Hence $\mu_{\infty}^{\tau_{p}}\left(x_{p}\right)=\mu_{\infty}(x)=0$. Applying now Proposition 1.2 to the element $x_{p} \in S_{0}^{+}\left(\mathcal{M}_{p}, \tau_{p}\right)$ and in view of $\tau_{p}\left(\mathbf{1}_{p}\right)=\tau(p)=\infty$, there exists a $*$ isomorphism $V$ from $S([0, \infty), m)$ into $S\left(\mathcal{M}_{p}, \tau\right)$ such that $V\left(\mu^{\tau_{p}}\left(x_{p}\right)\right)=x_{p}$ and $\mu^{\tau_{p}}(V(f))=\mu(f)$ for all $f \in S([0, \infty), m)$. The function $\Psi_{p}\left(y_{p}\right)=$ $\Phi_{p}^{-1}\left(y_{p}\right)=$ pyp for $y_{p} \in S\left(\mathcal{M}_{p}, \tau_{p}\right)$ is a $*$-isomorphism from $S\left(\mathcal{M}_{p}, \tau_{p}\right)$ onto $p S(\mathcal{M}, \tau) p$.

Let $W=\Psi_{p} \circ V$; then $W$ is a $*$-isomorphism from $S([0, \infty), m)$ into $p S(\mathcal{M}, \tau) p$. Since $p x p=x, \mu(x)=\mu^{\tau_{p}}\left(x_{p}\right)$, we have

$$
W(\mu(x))=\Psi_{p}(V(\mu(x)))=\Psi_{p}\left(V\left(\mu^{\tau_{p}}\left(x_{p}\right)\right)\right)=\Psi_{p}\left(x_{p}\right)=p x p=x .
$$

Note that for any $y \in S\left(\mathcal{M}_{p}, \tau_{p}\right), p y=y$ and $y=\left.y p\right|_{p(H)}=(y p)_{p}$. Finally, since $V(f) \in S\left(\mathcal{M}_{p}, \tau_{p}\right)$,

$$
\begin{aligned}
\mu(W(f)) & =\mu\left(\Psi_{p}(V(f))\right)=\mu\left(\Psi_{p}\left((V(f) p)_{p}\right)\right)=\mu(p V(f) p) \\
& =\mu(p|V(f)| p)=\mu(p|V(f)| p p)=\mu^{\tau_{p}}\left((|V(f)| p)_{p}\right) \\
& =\mu^{\tau_{p}}(|V(f)|)=\mu^{\tau_{p}}(V(f))=\mu(f),
\end{aligned}
$$

which finishes the proof.

Let $(X,\|\cdot\|)$ be a Banach space over the field of complex numbers. We denote by $B_{X}$ and $S_{X}$ the unit ball and the unit sphere in $X$, respectively. We now define several geometric properties of $X$ which will be studied in this paper.

We say that $x \in S_{X}$ is a strongly extreme point of the unit ball $B_{X}$, or $M L U R$ point of $B_{X}$ [29], if for any $\left\{y_{n}\right\},\left\{z_{n}\right\} \subset B_{X},\left\|2 x-y_{n}-z_{n}\right\| \rightarrow 0$ implies that $\left\|y_{n}-z_{n}\right\| \rightarrow 0$. Equivalently, $x \in S_{X}$ is a strongly extreme point if for any $\left\{y_{n}\right\} \subset X,\left\|x \pm y_{n}\right\| \rightarrow 1$ implies $\left\|y_{n}\right\| \rightarrow 0$. A Banach space $X$ is called midpoint locally uniformly rotund $(M L U R)$ if every element of $S_{X}$ is a strongly extreme point.

A point $x$ of $S_{X}$ is said to be a complex extreme point ( $\mathbb{C}$-extreme point) of the unit ball $B_{X}$ [35] if for every $\lambda \in \mathbb{C}$ with $|\lambda| \leq 1$ and $y$ in $X$, whenever $x+\lambda y \in B_{X}$ then $y=0$. Equivalently, $x$ is a complex extreme point for $B_{X}$ if whenever $x \pm y, x \pm i y \in B_{X}, y \in B_{X}$, then $y=0$. The 
space $X$ is said to be complex rotund ( $\mathbb{C}$-rotund) if every element of $S_{X}$ is a $\mathbb{C}$-extreme point. We denote by $\mathbb{C}$-ext $\left(B_{X}\right)$ the set of all complex extreme points of $B_{X}$.

A point $x \in S_{X}$ is a point of complex local uniform rotundity ( $\mathbb{C}-L U R$ point) [37] if for every $\epsilon>0$ there exists $\delta(x, \epsilon)>0$ such that

$$
\sup _{\lambda= \pm 1, \pm i}\|x+\lambda y\| \geq 1+\delta(x, \epsilon)
$$

for every $y \in X$ satisfying $\|y\| \geq \epsilon$. Equivalently, $x$ is a $\mathbb{C}$-LUR point if from $\left\|x+\lambda y_{n}\right\| \rightarrow 1,\left\{y_{n}\right\} \subset X, \lambda= \pm 1, \pm i$ it follows that $\left\|y_{n}\right\| \rightarrow 0$. If every point of $S_{X}$ is a $\mathbb{C}$-LUR point, then $X$ is called a complex locally uniformly rotund $(\mathbb{C}-L U R)$ space.

It is clear that real geometric properties such as uniform rotundity, local uniform rotundity and rotundity imply their complex analogues, that is, complex uniform rotundity, complex local uniform rotundity and complex rotundity, respectively.

There also exist the notions of complex strongly extreme points $(\mathbb{C}$ MLUR points) and $\mathbb{C}-M L U R$ spaces. They are defined analogously to MLUR points and MLUR spaces following the idea in [20]. However, as we will show in the last section, the notions of $\mathbb{C}$-LUR points and $\mathbb{C}$-MLUR points coincide in any complex Banach space, and consequently the $\mathbb{C}$-LUR and $\mathbb{C}$-MLUR properties are equivalent. We wish to point out that there exist $\mathbb{C}$-LUR spaces that are not complex uniformly rotund, and complex rotund spaces that are not $\mathbb{C}$-LUR. We will briefly discuss some examples in the last section.

It is well known that monotone properties of Banach lattices are closely related to their complex rotundity properties [23, 28]. The interplay between those properties is an important factor in investigating complex properties of Banach lattices. Let us recall some monotonicity notions employed further in this paper.

A point $f$ in a Banach lattice $\left(E,\|\cdot\|_{E}\right)$ is called upper monotone, or $U M$, if for any $g \in E$ the condition $g \geq f$ and $g \neq f$ implies that $\|g\|_{E}>\|f\|_{E}$.

An element $0 \leq f \in S_{E}$ is called upper locally uniformly monotone, or $U L U M$, if for any sequence $\left\{f_{n}\right\}$ such that $f \leq f_{n}$, if $\left\|f_{n}\right\|_{E} \rightarrow 1$ then $\left\|f_{n}-f\right\|_{E} \rightarrow 0$.

The following results relating monotonicity and complex rotundity properties for the Banach lattice $E$ appear very useful in our investigations.

THEOREM 1.4 ([23]).

(1) An element $f$ of a complex Banach lattice $E$ is a $\mathbb{C}$-extreme point if and only if $|f|$ is an $U M$ point in its real part $E_{r}$. 
(2) If $f$ is a $\mathbb{C}$-LUR point of a complex Banach lattice $E$, then $|f|$ is an $U L U M$ point in its real part $E_{r}$.

We finish this section with an elementary result on Banach spaces.

Lemma 1.5. Let $(X,\|\cdot\|)$ be a normed space. If $\left\{y_{n}\right\} \subset X,\|x\|=1$ and $\varlimsup_{n}\left\|x \pm y_{n}\right\| \leq 1$, then $\lim _{n}\left\|x \pm y_{n}\right\|=1$.

Proof. Since $\|x\|=1$ and $\varlimsup_{n}\left\|x \pm y_{n}\right\| \leq 1$, from the inequality

$$
2=2\|x\| \leq\left\|x+y_{n}\right\|+\left\|x-y_{n}\right\|
$$

it follows that $\varlimsup_{n}\left\|x \pm y_{n}\right\|=1$. Note that $1-\left\|x-y_{n}\right\| / 2 \leq\left\|x+y_{n}\right\| / 2$ and therefore

$$
1-\varlimsup_{n}\left\|x-y_{n}\right\| / 2 \leq \varliminf_{n}\left(1-\left\|x-y_{n}\right\| / 2\right) \leq \frac{\varliminf_{n}}{n}\left\|x+y_{n}\right\| / 2 .
$$

Since $\varlimsup_{n}\left\|x \pm y_{n}\right\|=1$, we get $1 \leq \underline{\lim }_{n}\left\|x+y_{n}\right\| \leq \varlimsup_{n}\left\|x+y_{n}\right\|=1$, and so $\lim _{n}\left\|x+y_{n}\right\|=1$. Similarly one can show that $\lim _{n}\left\|x-y_{n}\right\|=1$.

Convention. Throughout the paper, $E$ will stand for a symmetric function space on $[0, \alpha)$, where $\alpha=\tau(\mathbf{1})$. Also the semifinite von Neumann algebra $\mathcal{M}$ is always fixed and has a faithful, normal, semifinite trace $\tau$.

2. Strongly extreme points and midpoint local uniform rotundity. In this section we will discuss strongly extreme points and midpoint local uniform rotundity of $E(\mathcal{M}, \tau)$. We will first show that if $\mu(x)$ is an order continuous, strongly extreme point of the unit ball in $E$, then $x$ is a strongly extreme point of the unit ball in $E(\mathcal{M}, \tau)$. We shall need the following two lemmas. To ensure that $E$ is an interpolation space between $L^{1}$ and $L^{\infty}$ we need to assume that $E$ is a fully symmetric space [3, 27].

Lemma 2.1. Suppose that $E$ is fully symmetric and $\mu(x)$ is a MLUR point of $B_{E}$. Then for any $\left\{y_{n}\right\},\left\{z_{n}\right\} \subset B_{E(\mathcal{M}, \tau)}$, if $\left\|2 x-y_{n}-z_{n}\right\| \rightarrow 0$ then $\left\|\mu(x)-\mu\left(y_{n}\right)\right\| \rightarrow 0$ and $\left\|\mu(x)-\mu\left(z_{n}\right)\right\| \rightarrow 0$.

Proof. Suppose that $\left\|2 x-y_{n}-z_{n}\right\|_{E(\mathcal{M}, \tau)} \rightarrow 0,\left\{y_{n}\right\},\left\{z_{n}\right\} \subset B_{E(\mathcal{M}, \tau)}$. Note that $\mu(2 x) \prec \mu\left(2 x-y_{n}-z_{n}\right)+\mu\left(y_{n}\right)+\mu\left(z_{n}\right)$. By [3․ Chapter 3, Theorems 2.10 and 2.12] and [27, Chapter 2, Theorem 4.3], for any $n \in \mathbb{N}$, there exists a substochastic linear operator $T_{n}: E \rightarrow E$ such that

$$
T_{n} \mu\left(2 x-y_{n}-z_{n}\right)+T_{n} \mu\left(y_{n}\right)+T_{n} \mu\left(z_{n}\right)=\mu(2 x) .
$$

Therefore

$$
\left\|\mu(2 x)-T_{n} \mu\left(y_{n}\right)-T_{n} \mu\left(z_{n}\right)\right\|_{E}=\left\|T_{n} \mu\left(2 x-y_{n}-z_{n}\right)\right\|_{E} \leq\left\|2 x-y_{n}-z_{n}\right\|_{E(\mathcal{M}, \tau)},
$$


which implies that $\left\|2 \mu(x)-T_{n} \mu\left(y_{n}\right)-T_{n} \mu\left(z_{n}\right)\right\|_{E} \rightarrow 0$. Applying the fact that $\mu(x)$ is a MLUR point of $B_{E}$, we get

$$
\left\|\mu(x)-T_{n} \mu\left(y_{n}\right)\right\|_{E} \rightarrow 0 \text { and }\left\|\mu(x)-T_{n} \mu\left(z_{n}\right)\right\|_{E} \rightarrow 0 .
$$

It remains to show that $\left\|T_{n} \mu\left(y_{n}\right)-\mu\left(y_{n}\right)\right\|_{E} \rightarrow 0$ and $\left\|T_{n} \mu\left(z_{n}\right)-\mu\left(z_{n}\right)\right\|_{E}$ $\rightarrow 0$. Define $f_{n}=T_{n} \mu\left(y_{n}\right) / 2-\mu\left(y_{n}\right) / 2$. For every $n \in \mathbb{N}$,

$$
\begin{aligned}
\left\|\mu(x)-f_{n}\right\|_{E} & =\left\|\mu(x)-T_{n} \mu\left(y_{n}\right) / 2+\mu\left(y_{n}\right) / 2\right\|_{E} \\
& \leq\left\|\mu(x)-T_{n} \mu\left(y_{n}\right)\right\|_{E}+\left\|T_{n} \mu\left(y_{n}\right)\right\|_{E} / 2+\left\|\mu\left(y_{n}\right)\right\|_{E} / 2 \\
& \leq\left\|\mu(x)-T_{n} \mu\left(y_{n}\right)\right\|_{E}+1,
\end{aligned}
$$

and in view of $\mu(x)-\frac{1}{2} \mu(y) \prec \mu\left(x-\frac{1}{2} y\right)$,

$$
\begin{aligned}
\left\|\mu(x)+f_{n}\right\|_{E} & =\left\|\mu(x)+T_{n} \mu\left(y_{n}\right) / 2-\mu\left(y_{n}\right) / 2\right\|_{E} \\
& \leq\left\|\mu(x)-\mu\left(y_{n} / 2\right)\right\|_{E}+\left\|T_{n} \mu\left(y_{n}\right)\right\|_{E} / 2 \\
& \leq\left\|x-y_{n} / 2\right\|_{E(\mathcal{M}, \tau)}+\left\|y_{n}\right\|_{E(\mathcal{M}, \tau)} / 2 \\
& \leq\left\|x-y_{n} / 2-z_{n} / 2\right\|_{E(\mathcal{M}, \tau)}+\left\|y_{n}\right\|_{E(\mathcal{M}, \tau)} / 2+\left\|z_{n}\right\|_{E(\mathcal{M}, \tau)} / 2 \\
& \leq\left\|x-y_{n} / 2-z_{n} / 2\right\|_{E(\mathcal{M}, \tau)}+1 .
\end{aligned}
$$

Consequently,

$$
\varlimsup_{n}\left\|\mu(x) \pm f_{n}\right\|_{E} \leq 1 .
$$

Now by Lemma 1.5 we have $\lim _{n}\left\|\mu(x) \pm f_{n}\right\|_{E}=1$. As $\mu(x)$ is a MLUR point, we deduce that $\left\|f_{n}\right\|_{E}=\frac{1}{2}\left\|T_{n} \mu\left(y_{n}\right)-\mu\left(y_{n}\right)\right\|_{E} \rightarrow 0$. Similarly, one can show that $\left\|T_{n} \mu\left(z_{n}\right)-\mu\left(z_{n}\right)\right\|_{E} \rightarrow 0$.

Let $\mathcal{N}=\left\{N_{f}: L^{2}(0,1) \rightarrow L^{2}(0,1): f \in L^{\infty}(0,1)\right\}$ be a commutative von Neumann algebra with the trace $\eta\left(N_{f}\right)=\int_{0}^{1} f d m$, where $m$ is the Lebesgue measure on $[0,1]$. Let $\mathcal{A}=\mathcal{N} \bar{\otimes} \mathcal{M}$ be the tensor product of the von Neumann algebras $\mathcal{N}$ and $\mathcal{M}$, and $\kappa=\eta \otimes \tau$ be the tensor product of the traces $\eta$ and $\tau$, that is, $\kappa\left(N_{f} \otimes x\right)=\eta\left(N_{f}\right) \tau(x)$, [24, 34]. It is well known that $\mathcal{A}$ has no atoms.

Let $\mathbb{1}$ be the identity operator in $L^{2}(0,1)$ and set $\mathbb{C} \mathbb{1}=\{\lambda \mathbb{1}: \lambda \in \mathbb{C}\}$. Let $x \in S(\mathcal{M}, \tau)$ and consider a linear subspace $D$ in $L^{2}(0,1) \otimes H$ generated by the vectors of the form $\zeta \otimes \xi$, where $\zeta \in L^{2}(0,1)$ and $\xi \in \mathcal{D}(x) \subset H$. For every $\xi=\sum_{i=1}^{n} \zeta_{i} \otimes \xi_{i} \in D$ define $(\mathbb{1} \otimes x)(\xi)=\sum_{i=1}^{n} \zeta_{i} \otimes x \xi_{i}$. The linear operator $\mathbb{1} \otimes x: D \rightarrow L^{2}(0,1) \otimes H$ with domain $D$ is preclosed and its closure $\mathbb{1} \otimes x$ is contained in $S(\mathbb{C} \mathbb{1} \otimes \mathcal{M}, \kappa)[18,31,32$.

The map $\pi: x \mapsto \mathbb{1} \otimes x, x \in \mathcal{M}$, is a unital trace-preserving *isomorphism from $\mathcal{M}$ onto the von Neumann subalgebra $\mathbb{C} \mathbb{1} \otimes \mathcal{M}$. Consequently, $\pi$ extends uniquely to a $*$-isomorphism $\tilde{\pi}$ from $S(\mathcal{M}, \tau)$ onto $S(\mathbb{C} \mathbb{1} \otimes \mathcal{M}, \kappa)$. In fact one can show that $\tilde{\pi}(x)=\mathbb{1} \bar{\otimes} x$.

Moreover $\tilde{\pi}$ preserves the singular value function in the sense that $\tilde{\mu}(\tilde{\pi}(x))$ $=\mu(x)$, where $\tilde{\mu}(\tilde{\pi}(x))$ is the singular value function of $\tilde{\pi}(x)$ computed with respect to the von Neumann algebra $\mathbb{C} \mathbb{1} \otimes \mathcal{M}$ and the trace $\kappa$. 
Thus

$$
\begin{aligned}
E(\mathbb{C} \mathbb{1} \otimes \mathcal{M}, \kappa) & =\{y \in S(\mathbb{C} \mathbb{1} \otimes \mathcal{M}, \kappa): \tilde{\mu}(y) \in E\} \\
& =\{\tilde{\pi}(x): x \in S(\mathcal{M}, \tau) \text { and } \mu(x) \in E\}
\end{aligned}
$$

where

$$
\|\tilde{\pi}(x)\|_{E(\mathbb{C} \mathbb{1} \otimes \mathcal{M}, \kappa)}=\|\tilde{\mu}(\tilde{\pi}(x))\|_{E}=\|\mu(x)\|_{E}=\|x\|_{E(\mathcal{M}, \tau)} .
$$

Hence $\tilde{\pi}$ is a $*$-isomorphism which is also an isometry from $E(\mathcal{M}, \tau)$ onto $E(\mathbb{C} \mathbb{1} \otimes \mathcal{M}, \kappa)$. We refer the reader to [18] for the details.

With this preparation we can prove the next result for any von Neumann algebra. For nonatomic von Neumann algebras it was shown implicitly in the proof of Theorem 2.1 in [6].

Lemma 2.2. For any $x, x_{n} \in E_{0}(\mathcal{M}, \tau), n \in \mathbb{N}$, we have $x_{n} \stackrel{\tau}{\rightarrow} x$ whenever $\left\|\mu(x)-\mu\left(x_{n}\right)\right\|_{E} \rightarrow 0$ and $\left\|\mu(x)-\mu\left(\left(x+x_{n}\right) / 2\right)\right\|_{E} \rightarrow 0$.

Proof. Suppose that $x_{n} \in E_{0}(\mathcal{M}, \tau), n \in \mathbb{N},\left\|\mu(x)-\mu\left(x_{n}\right)\right\|_{E} \rightarrow 0$ and $\left\|\mu(x)-\mu\left(\left(x+x_{n}\right) / 2\right)\right\|_{E} \rightarrow 0$. In view of the assumptions and the remarks preceding the lemma,

and

$$
\left\|\tilde{\mu}(\mathbb{1} \bar{\otimes} x)-\tilde{\mu}\left(\mathbb{1} \bar{\otimes} x_{n}\right)\right\|_{E} \rightarrow 0
$$

$\left\|\tilde{\mu}(\mathbb{1} \bar{\otimes} x)-\tilde{\mu}\left(\left(\mathbb{1} \bar{\otimes} x+\mathbb{1} \bar{\otimes} x_{n}\right) / 2\right)\right\|_{E}=\left\|\tilde{\mu}(\mathbb{1} \bar{\otimes} x)-\tilde{\mu}\left(\mathbb{1} \bar{\otimes}\left(\left(x+x_{n}\right) / 2\right)\right)\right\|_{E} \rightarrow 0$.

Since $\mathbb{C} \mathbb{1} \otimes \mathcal{M}$ is nonatomic it follows that $\mathbb{1} \bar{\otimes} x-\mathbb{1} \bar{\otimes} x_{n} \stackrel{\kappa}{\rightarrow} 0$. The latter is equivalent to the fact that for a.e. $t>0, \tilde{\mu}_{t}\left(\mathbb{1} \bar{\otimes} x-\mathbb{1} \bar{\otimes} x_{n}\right) \rightarrow 0$. Thus for a.e. $t>0$,

$$
\mu_{t}\left(x-x_{n}\right)=\tilde{\mu}_{t}\left(\mathbb{1} \bar{\otimes}\left(x-x_{n}\right)\right)=\tilde{\mu}_{t}\left(\mathbb{1} \bar{\otimes} x-\mathbb{1} \bar{\otimes} x_{n}\right) \rightarrow 0
$$

and so $x-x_{n} \stackrel{\tau}{\rightarrow} 0$.

Before we state the first main theorem of this section we need a few facts about order continuous elements of $E(\mathcal{M}, \tau)$. The next proposition relates the order continuity of an operator $x$ to the order continuity of its singular value function.

Proposition 2.3. An operator $x \in E(\mathcal{M}, \tau)$ is order continuous if and only if $\mu(x)$ is order continuous in $E$. Therefore $E(\mathcal{M}, \tau)^{\mathrm{oc}}=E^{\mathrm{oc}}(\mathcal{M}, \tau)$.

Proof. Suppose that $\mu(x)$ is order continuous in $E$ and $0 \downarrow_{n} x_{n} \leq|x|$. Then $\mu_{\infty}(x)=0$ and by [14, Lemma 3.5], $\mu_{t}\left(x_{n}\right) \downarrow_{n} 0$ for all $t>0$. Therefore $\left\|x_{n}\right\|_{E(\mathcal{M}, \tau)}=\left\|\mu\left(x_{n}\right)\right\|_{E} \rightarrow 0$ and $x$ is an order continuous element of $E(\mathcal{M}, \tau)$.

Let now $x$ be an order continuous element of $E(\mathcal{M}, \tau)$. Hence $\mu_{\infty}(x)=0$ and for $p=s(x) \vee s\left(x^{*}\right)$ the trace $\tau_{p}$ on $\mathcal{M}_{p}$ is $\sigma$-finite. It is easy to show that $x_{p}$ is an order continuous element of $E\left(\mathcal{M}_{p}, \tau_{p}\right)$. Indeed, let $\left\{y_{n}\right\} \subset$ $E\left(\mathcal{M}_{p}, \tau_{p}\right)$ be such that $0 \downarrow_{n} y_{n} \leq\left|x_{p}\right|=|x|_{p}$. Then for all $n \in \mathbb{N}, y_{n}=\left(x_{n}\right)_{p}$ 
for some positive $x_{n} \in E(\mathcal{M}, \tau)$. Moreover, from $0 \downarrow_{n}\left(x_{n}\right)_{p} \leq|x|_{p}$ it follows that $0 \downarrow_{n} p x_{n} p \leq p|x| p \leq|x|$. As $x$ is order continuous, $\left\|y_{n}\right\|_{E\left(\mathcal{M}_{p}, \tau_{p}\right)}=$ $\left\|p x_{n} p\right\|_{E(\mathcal{M}, \tau)} \rightarrow 0$, proving that $x_{p}$ is order continuous. Moreover $\mu^{\tau_{p}}\left(x_{p}\right)=$ $\mu(p x p)=\mu(x)$, where $\mu^{\tau_{p}}$ is the singular value function computed with respect to the trace $\tau_{p}$ and the von Neumann algebra $\mathcal{M}_{p}$. Therefore we can assume that the trace $\tau$ is $\sigma$-finite. Consider a $*$-isomorphism $\tilde{\pi}$ from $E(\mathcal{M}, \tau)$ onto $E(\mathbb{C} \mathbb{1} \otimes \mathcal{M}, \kappa)$, where $\mathbb{C} \mathbb{1} \otimes \mathcal{M}$ is a nonatomic von Neumann algebra. It is not difficult to see that $x$ is order continuous in $E(\mathcal{M}, \tau)$ if and only if $\tilde{\pi}(x)$ is order continuous in $E(\mathbb{C} \mathbb{1} \otimes \mathcal{M}, \kappa)$. Since for the singular value function $\tilde{\mu}(\tilde{\pi}(x))$ of $\tilde{\pi}(x)$ computed with respect to the von Neumann algebra $\mathbb{C} \mathbb{1} \otimes \mathcal{M}$ and the trace $\kappa$, we have $\tilde{\mu}(\tilde{\pi}(x))=\mu(x)$, it can be assumed that the von Neumann algebra $\mathcal{M}$ is nonatomic.

Suppose that a.e. $0 \downarrow_{n} f_{n} \leq \mu(x)$. By Proposition 1.2, there exists a *-isomorphism $V$ from $S([0, \tau(\mathbf{1})), m)$ into $S(\mathcal{M}, \tau)$ such that $V(\mu(x))=|x|$ and $\mu(V(f))=\mu(f)$ for all $f \in S([0, \tau(\mathbf{1})), m)$. Therefore $0 \downarrow_{n} V\left(f_{n}\right) \leq$ $V(\mu(x))=|x|$ and since $x$ is order continuous, $\left\|f_{n}\right\|_{E}=\left\|V\left(f_{n}\right)\right\|_{E(\mathcal{M}, \tau)} \rightarrow 0$.

The following convergence result is known under the stronger assumption that the whole space $E$ is order continuous [6, Proposition 1.1].

Proposition 2.4. Let $E$ be strongly symmetric. For an order continuous element $x \in E(\mathcal{M}, \tau)$ and $\left\{x_{n}\right\} \subset E(\mathcal{M}, \tau)$, the following conditions are equivalent:

(i) $\left\|x-x_{n}\right\|_{E(\mathcal{M}, \tau)} \rightarrow 0$,

(ii) $\left\|\mu(x)-\mu\left(x_{n}\right)\right\|_{E} \rightarrow 0$ and $x_{n} \stackrel{\tau}{\rightarrow} x$.

Proof. Since $\mu(x)-\mu\left(x_{n}\right) \prec \mu\left(x-x_{n}\right), n \in \mathbb{N}$, and the embedding of $E(\mathcal{M}, \tau)$ in $S(\mathcal{M}, \tau)$ is continuous, (i) implies (ii).

Suppose now that (ii) holds. Note that it is enough to show that there exists a subsequence that satisfies (i), since this implies that every subsequence has a subsequence which satisfies (i). If $x_{n}-x \stackrel{\tau}{\rightarrow} 0$, then by [18, Chapter II, Lemma 5.15], passing to a subsequence of $\left\{x_{n}\right\}$, there exists a sequence $\left\{p_{j}\right\} \subset \mathcal{P}(\mathcal{M})$ satisfying $p_{j} \uparrow \mathbf{1}, \tau\left(p_{j}^{\perp}\right) \rightarrow 0$ as $j \rightarrow \infty$ and $\left\|\left(x-x_{n}\right) p_{j}\right\|_{\mathcal{M}} \rightarrow 0$ as $n \rightarrow \infty$ for all $j \in \mathbb{N}$.

For all projections $p$ in $E(\mathcal{M}, \tau)$ and all $n, j \in \mathbb{N}$ we have

$$
\begin{aligned}
& \left\|\left(x-x_{n}\right) p\right\|_{E(\mathcal{M}, \tau)} \leq\left\|\left(x-x_{n}\right) p_{j} p\right\|_{E(\mathcal{M}, \tau)}+\left\|\left(x-x_{n}\right) p_{j}^{\perp} p\right\|_{E(\mathcal{M}, \tau)} \\
& \leq\left\|\left(x-x_{n}\right) p_{j}\right\|_{\mathcal{M}}\|p\|_{E(\mathcal{M}, \tau)}+\left\|\mu(x) \chi_{\left[0, \tau\left(p_{j}^{\perp}\right)\right)}+\mu\left(x_{n}\right) \chi_{\left[0, \tau\left(p_{j}^{\perp}\right)\right)}\right\|_{E} \\
& \leq\left\|\left(x-x_{n}\right) p_{j}\right\|_{\mathcal{M}}\|p\|_{E(\mathcal{M}, \tau)}+\left\|\mu\left(x_{n}\right) \chi_{\left[0, \tau\left(p_{j}^{\perp}\right)\right)}-\mu(x) \chi_{\left[0, \tau\left(p_{j}^{\perp}\right)\right)}\right\|_{E} \\
& \quad+2\left\|\mu(x) \chi_{\left[0, \tau\left(p_{j}^{\perp}\right)\right)}\right\|_{E} \\
& \leq\left\|\left(x-x_{n}\right) p_{j}\right\|_{\mathcal{M}}\|p\|_{E(\mathcal{M}, \tau)}+\left\|\mu\left(x_{n}\right)-\mu(x)\right\|_{E}+2\left\|\mu(x) \chi_{\left[0, \tau\left(p_{j}^{\perp}\right)\right)}\right\|_{E} .
\end{aligned}
$$


Consider first the case when $x_{n} \in S_{0}(\mathcal{M}, \tau)$ for all $n \in \mathbb{N}$. Since $x$ is order continuous, also $x \in S_{0}(\mathcal{M}, \tau)$. Set $q(x)=s(x) \vee s\left(x^{*}\right)$ and $q\left(x_{n}\right)=$ $s\left(x_{n}\right) \vee s\left(x_{n}^{*}\right), n \in \mathbb{N}$, and define $p=\vee_{n=1}^{\infty} q\left(x_{n}\right) \vee q(x)$. Then the trace $\tau_{p}$ is $\sigma$-finite on the von Neumann algebra $\mathcal{M}_{p}$. Moreover, for all $n \in \mathbb{N}$, $\left\|x_{p}-\left(x_{n}\right)_{p}\right\|_{E\left(\mathcal{M}_{p}, \tau_{p}\right)}=\left\|p\left(x-x_{n}\right) p\right\|_{E(\mathcal{M}, \tau)}=\left\|x-x_{n}\right\|_{E(\mathcal{M}, \tau)}, \mu^{\tau_{p}}\left(\left(x_{n}\right)_{p}\right)=$ $\mu\left(x_{n}\right), \mu^{\tau_{p}}\left(x_{p}\right)=\mu(x)$ and $\mu^{\tau_{p}}\left(x_{p}-\left(x_{n}\right)_{p}\right)=\mu\left(x-x_{n}\right)$, where $\mu^{\tau_{p}}$ is the singular value function computed with respect to the trace $\tau_{p}$ and $\mathcal{M}_{p}$. Therefore we can assume that the trace $\tau$ is $\sigma$-finite. Consider the nonatomic von Neumann algebra $\mathbb{C} \mathbb{1} \otimes \mathcal{M}$ and the $*$-isomorphism $\tilde{\pi}: x \mapsto \mathbb{1} \bar{\otimes} x$ from $S(\mathcal{M}, \tau)$ onto $S(\mathbb{C} \mathbb{1} \otimes \mathcal{M}, \kappa)$. Since $\tilde{\pi}$ preserves the singular value function, it can also be assumed that the von Neumann algebra $\mathcal{M}$ is nonatomic.

Let $\left\{e_{k}\right\} \subset \mathcal{P}(\mathcal{M})$ be such that $e_{k} \uparrow_{k} \mathbf{1}$ and $\tau\left(e_{k}\right)<\infty$ for all $k \in \mathbb{N}$. By Proposition 1.2, for each $n \in \mathbb{N}$ there exists a $*$-isomorphism $V_{n}$ from $S([0, \tau(\mathbf{1})), m)$ into $S(\mathcal{M}, \tau)$ such that $V_{n}\left(\mu\left(x_{n}\right)\right)=\left|x_{n}\right|$ and $\mu\left(V_{n}(f)\right)=$ $\mu(f)$ for all $f \in S([0, \tau(\mathbf{1})), m)$. Let $q_{n k}=V_{n}\left(\chi_{\left[0, \tau\left(e_{k}\right)\right)}\right), n, k \in \mathbb{N}$. Clearly, each $q_{n k}$ is a projection with $\tau\left(q_{n k}\right)=\tau\left(e_{k}\right)<\infty$, and therefore $q_{n k} \in$ $E(\mathcal{M}, \tau)$. Since $\left|x_{n} q_{n k}^{\perp}\right|=|| x_{n}\left|q_{n k}^{\perp}\right|$ and $q_{n k}^{\perp}=\mathbf{1}-q_{n k}=V_{n}\left(\chi_{[0, \tau(\mathbf{1}))}\right)-$ $V_{n}\left(\chi_{\left[0, \tau\left(e_{k}\right)\right)}\right)=V_{n}\left(\chi_{\left[\tau\left(e_{k}\right), \tau(\mathbf{1})\right)}\right)$, for all $n, k \in \mathbb{N}$ we have

$$
\begin{aligned}
& \left\|x_{n} q_{n k}^{\perp}\right\|_{E(\mathcal{M}, \tau)}=\left\|\left|x_{n}\right| q_{n k}^{\perp}\right\|_{E(\mathcal{M}, \tau)} \\
& \quad=\left\|V_{n}\left(\mu\left(x_{n}\right)\right) V_{n}\left(\chi_{\left[\tau\left(e_{k}\right), \tau(\mathbf{1})\right)}\right)\right\|_{E(\mathcal{M}, \tau)}=\left\|\mu\left(x_{n}\right) \chi_{\left[\tau\left(e_{k}\right), \tau(\mathbf{1})\right)}\right\|_{E} \\
& \quad \leq\left\|\mu\left(x_{n}\right) \chi_{\left[\tau\left(e_{k}\right), \tau(\mathbf{1})\right)}-\mu(x) \chi_{\left[\tau\left(e_{k}\right), \tau(\mathbf{1})\right)}\right\|_{E}\left\|\mu(x) \chi_{\left[\tau\left(e_{k}\right), \tau(\mathbf{1})\right)}\right\|_{E} \\
& \quad \leq\left\|\mu\left(x_{n}\right)-\mu(x)\right\|_{E}+\left\|\mu(x) \chi_{\left[\tau\left(e_{k}\right), \tau(\mathbf{1})\right)}\right\|_{E} .
\end{aligned}
$$

Let $\epsilon>0$. Since $x$ and $\mu(x)$ are order continuous by Proposition 2.3 . $\left\|x e_{k}^{\perp}\right\|_{E(\mathcal{M}, \tau)} \rightarrow 0,\left\|\mu(x) \chi_{\left[\tau\left(e_{k}\right), \tau(\mathbf{1})\right)}\right\|_{E} \rightarrow 0$ and $\left\|\mu(x) \chi_{\left[0, \tau\left(p_{j}^{\perp}\right)\right)}\right\|_{E} \rightarrow 0$ as $k, j \rightarrow \infty$. Therefore there exist $k_{0}, j_{0} \in \mathbb{N}$ such that $\left\|x e_{k_{0}}^{\perp}\right\|_{E(\mathcal{M}, \tau)} \leq \epsilon / 5$, $\left\|\mu(x) \chi_{\left[\tau\left(e_{k_{0}}\right), \tau(\mathbf{1})\right)}\right\|_{E} \leq \epsilon / 5$ and $\left\|\mu(x) \chi_{\left[0, \tau\left(p_{j_{0}}^{\perp}\right)\right)}\right\|_{E} \leq \epsilon / 20$. Observe that $\left\|q_{n k}\right\|_{E(\mathcal{M}, \tau)}=\left\|V_{n}\left(\chi_{\left[0, \tau\left(e_{k}\right)\right)}\right)\right\|_{E(\mathcal{M}, \tau)}=\left\|\chi_{\left[0, \tau\left(e_{k}\right)\right)}\right\|_{E}=\left\|e_{k}\right\|_{E(\mathcal{M}, \tau)}$ and $\mathbf{1}=$ $q_{n k}+q_{n k}^{\perp}=q_{n k}+q_{n k}^{\perp}\left(e_{k}+e_{k}^{\perp}\right)=q_{n k}+q_{n k}^{\perp} e_{k}+q_{n k}^{\perp} e_{k}^{\perp}, n, k \in \mathbb{N}$. This, combined with inequalities (2.1) and (2.2), implies that for all $n \in \mathbb{N}$,

$$
\begin{aligned}
\left\|x-x_{n}\right\|_{E(\mathcal{M}, \tau) \leq} \leq & \left\|\left(x-x_{n}\right) q_{n k_{0}}\right\|_{E(\mathcal{M}, \tau)}+\left\|\left(x-x_{n}\right) q_{n k_{0}}^{\perp} e_{k_{0}}\right\|_{E(\mathcal{M}, \tau)} \\
& +\left\|x q_{n k_{0}}^{\perp} e_{k_{0}}^{\perp}\right\|_{E(\mathcal{M}, \tau)}+\left\|x_{n} q_{n k_{0}}^{\perp} e_{k_{0}}^{\perp}\right\|_{E(\mathcal{M}, \tau)} \\
\leq & \left\|\left(x-x_{n}\right) q_{n k_{0}}\right\|_{E(\mathcal{M}, \tau)}+\left\|\left(x-x_{n}\right) e_{k_{0}}\right\|_{E(\mathcal{M}, \tau)} \\
& +\left\|x e_{k_{0}}^{\perp}\right\|_{E(\mathcal{M}, \tau)}+\left\|x_{n} q_{n k_{0}}^{\perp}\right\|_{E(\mathcal{M}, \tau)} \\
\leq & 2\left\|\left(x-x_{n}\right) p_{j_{0}}\right\|_{\mathcal{M}}\left\|e_{k_{0}}\right\|_{E(\mathcal{M}, \tau)}+3\left\|\mu\left(x_{n}\right)-\mu(x)\right\|_{E}+3 \epsilon / 5 .
\end{aligned}
$$

Since $\left\|\left(x-x_{n}\right) p_{j_{0}}\right\|_{\mathcal{M}} \rightarrow 0$ and $\left\|\mu\left(x_{n}\right)-\mu(x)\right\|_{E} \rightarrow 0$ as $n \rightarrow \infty$, there exists $N \in \mathbb{N}$ such that for all $n \geq N,\left\|\left(x-x_{n}\right) p_{j_{0}}\right\|_{\mathcal{M}} \leq \epsilon /\left(10\left\|e_{k_{0}}\right\|_{E(\mathcal{M}, \tau)}\right)$ 
and $\left\|\mu\left(x_{n}\right)-\mu(x)\right\|_{E} \leq \epsilon / 15$. Consequently, $\left\|x-x_{n}\right\|_{E(\mathcal{M}, \tau)} \leq \epsilon$ for all $n \geq N$.

Suppose now that for some $n \in \mathbb{N}, \mu_{\infty}\left(x_{n}\right)>0$. Then $\mu\left(x_{n}\right)>c \chi_{[0, \tau(\mathbf{1}))}$ for some constant $c>0$ and therefore $\mu(\mathbf{1})=\chi_{[0, \tau(\mathbf{1}))} \in E$, which implies that $\mathbf{1} \in E(\mathcal{M}, \tau)$. By (2.1), it follows that

$$
\begin{aligned}
\left\|x-x_{n}\right\|_{E(\mathcal{M}, \tau)} \leq & \left\|\left(x-x_{n}\right) p_{j}\right\|_{\mathcal{M}}\|\mathbf{1}\|_{E(\mathcal{M}, \tau)}+\left\|\mu\left(x_{n}\right)-\mu(x)\right\|_{E} \\
& +2\left\|\mu(x) \chi_{\left[0, \tau\left(p_{j}^{\perp}\right)\right)}\right\|_{E}
\end{aligned}
$$

for all $n, j \in \mathbb{N}$. Therefore similarly to the previous case, one can show that $\left\|x-x_{n}\right\|_{E(\mathcal{M}, \tau)} \rightarrow 0$ as $n \rightarrow \infty$, and the claim follows.

THEOREM 2.5. Let $E$ be fully symmetric and $x$ be an order continuous element of $E(\mathcal{M}, \tau)$. If the singular value function $\mu(x)$ is a MLUR point of $B_{E_{0}}$ then $x$ is a MLUR point of $B_{E_{0}(\mathcal{M}, \tau)}$.

Proof. Let $\mu(x) \in S_{E_{0}}$ be a MLUR point of $B_{E_{0}}$, and suppose that we have $\left\|2 x-y_{n}-z_{n}\right\|_{E(\mathcal{M}, \tau)} \rightarrow 0,\left\{y_{n}\right\},\left\{z_{n}\right\} \subset B_{E_{0}(\mathcal{M}, \tau)}$. By Lemma 2.1.

$$
\left\|\mu(x)-\mu\left(y_{n}\right)\right\|_{E} \rightarrow 0 \quad \text { and } \quad\left\|\mu(x)-\mu\left(z_{n}\right)\right\|_{E} \rightarrow 0 .
$$

Also

$$
\left\|2 x-\left(y_{n}+x\right) / 2-\left(z_{n}+x\right) / 2\right\|_{E(\mathcal{M}, \tau)}=\left\|x-y_{n} / 2-z_{n} / 2\right\|_{E(\mathcal{M}, \tau)} \rightarrow 0,
$$

with $\left(y_{n}+x\right) / 2,\left(z_{n}+x\right) / 2$ in the unit ball of $E_{0}(\mathcal{M}, \tau)$. Again, referring to Lemma 2.1 we get

$$
\left\|\mu(x)-\mu\left(\left(y_{n}+x\right) / 2\right)\right\|_{E} \rightarrow 0 \quad \text { and } \quad\left\|\mu(x)-\mu\left(\left(z_{n}+x\right) / 2\right)\right\|_{E} \rightarrow 0 .
$$

Now, Lemma 2.2 yields $y_{n} \stackrel{\tau}{\rightarrow} x$ and $z_{n} \stackrel{\tau}{\rightarrow} x$. Thus $\left\|x-y_{n}\right\|_{E(\mathcal{M}, \tau)} \rightarrow 0$ and $\left\|x-z_{n}\right\|_{E(\mathcal{M}, \tau)} \rightarrow 0$ by Proposition 2.4 and $x$ is a MLUR point of $B_{E_{0}(\mathcal{M}, \tau)}$.

Next, we want to establish that if $x \in S_{E(\mathcal{M}, \tau)}$ is strongly extreme, then $\mu(x)$ is a strongly extreme point of $B_{E}$. We will need the following elementary lemma.

Lemma 2.6. Let $x \in S(\mathcal{M}, \tau)$. If $n(x) \sim n\left(x^{*}\right)$, then there exists an isometry $w$ such that $x=w|x|$.

Proof. Suppose that $n(x) \sim n\left(x^{*}\right)$, that is, $n(x)=v^{*} v$ and $n\left(x^{*}\right)=v v^{*}$, where $v$ is a partial isometry. Let $x=u|x|$ be a polar decomposition of $x$, that is, $u$ is a partial isometry with $\operatorname{Ker} u=\operatorname{Ker} x$. Set $w=u+v$. We claim that $w$ is an isometry, that is, $w^{*} w=\mathbf{1}$. To see this, note first that since Ker $u^{*}=\operatorname{Ker} x^{*}, u^{*} v v^{*}=u^{*} n\left(x^{*}\right)=0$. Thus $\left|v^{*} u\right|^{2}=u^{*} v v^{*} u=0$ and $v^{*} u=0$. Hence

$$
\begin{aligned}
w^{*} w & =\left(u^{*}+v^{*}\right)(u+v)=u^{*} u+u^{*} v+v^{*} u+v^{*} v \\
& =u^{*} u+\left(v^{*} u\right)^{*}+v^{*} u+v^{*} v=s(x)+n(x)=\mathbf{1},
\end{aligned}
$$

proving that $w$ is an isometry. 
Since $v^{*} v$ is a projection on $\mathrm{Ker}^{\perp} v$ and $0=(|x| n(x))^{*}=n(x)|x|=$ $v^{*} v|x|$, it follows that Range $|x| \subset \operatorname{Ker} v$. Consequently, $v|x|=0$. Therefore

$$
w|x|=(u+v)|x|=u|x|+v|x|=u|x|=x,
$$

and the proof is complete.

TheOREM 2.7. Suppose that the trace $\tau$ on $\mathcal{M}$ is $\sigma$-finite. If $x$ is a MLUR point of $B_{E(\mathcal{M}, \tau)}$ then $\mu(x)$ is a MLUR point of $B_{E}$ and either

(i) $\mu_{\infty}(x)=0$, or

(ii) $n(x) \mathcal{M} n\left(x^{*}\right)=0$ and $|x| \geq \mu_{\infty}(x) s(x)$.

Proof. Recall that there exists a $*$-isomorphism $\tilde{\pi}: x \mapsto \mathbb{1} \bar{\otimes} x$ from $S(\mathcal{M}, \tau)$ onto $S(\mathbb{C} \mathbb{1} \otimes \mathcal{M}, \kappa)$, which is also an isometry from $E(\mathcal{M}, \tau)$ onto $E(\mathbb{C} \mathbb{1} \otimes \mathcal{M}, \kappa)$. Hence $x$ is a MLUR point in $E(\mathcal{M}, \tau)$ if and only if $\mathbb{1} \bar{\otimes} x$ is a MLUR point in $E(\mathbb{C} \mathbb{1} \otimes \mathcal{M}, \kappa)$. Since also $\tilde{\mu}(\mathbb{1} \otimes x)=\mu(x)$, where $\tilde{\mu}$ is the singular value function computed with respect to the trace $\kappa$ and the nonatomic von Neumann algebra $\mathbb{C} \mathbb{1} \otimes \mathcal{M}$, without loss of generality we can assume that the von Neumann algebra $\mathcal{M}$ is nonatomic.

Suppose that $x$ is a MLUR point of the unit ball in $E(\mathcal{M}, \tau)$. Since every strongly extreme point is extreme, conditions (i) and (ii) are satisfied by the well known criterion on extreme points in $B_{E(\mathcal{M}, \tau)}$ [5]. It remains to show that $\mu(x)$ is a MLUR point of $B_{E}$.

Assume first that $\tau(s(x))=\infty$. By Proposition 1.1(2) and by (ii), we have $\mu\left(|x|-\mu_{\infty}(x) s(x)\right)=\mu(x)-\mu_{\infty}(x)$. Consequently, $\mu_{\infty}\left(|x|-\mu_{\infty}(x) s(x)\right)$ $=0$ and $|x|-\mu_{\infty}(x) s(x) \in S_{0}^{+}(\mathcal{M}, \tau)$. Also, in view of $s(x)|x|=|x| s(x)$ we have

$$
s(x)\left(|x|-\mu_{\infty}(x) s(x)\right)=\left(|x|-\mu_{\infty}(x) s(x)\right) s(x)=|x|-\mu_{\infty}(x) s(x) .
$$

Hence Proposition 1.3 applied to the element $|x|-\mu_{\infty}(x) s(x)$ with $p=s(x)$ yields a $*$-isomorphism $W$ from $S([0, \infty), m)$ into $s(x) S(\mathcal{M}, \tau) s(x)$ such that

$$
W\left(\mu\left(|x|-\mu_{\infty}(x) s(x)\right)\right)=|x|-\mu_{\infty}(x) s(x) \quad \text { and } \quad \mu(W(f))=\mu(f)
$$

for all $f \in S([0, \infty), m)$. As $W(1)=s(x)$, where $1=\chi_{[0, \infty)}$, it follows that

$$
\begin{aligned}
|x|-\mu_{\infty}(x) s(x) & =W\left(\mu\left(|x|-\mu_{\infty}(x) s(x)\right)\right)=W\left(\mu(x)-\mu_{\infty}(x)\right) \\
& =W(\mu(x))-\mu_{\infty}(x) W(1)=W(\mu(x))-\mu_{\infty}(x) s(x),
\end{aligned}
$$

and consequently $W(\mu(x))=|x|$.

Let $\left\|\mu(x)+\lambda f_{n}\right\|_{E} \rightarrow 1$ for $\lambda= \pm 1$, where $f_{n} \in B_{E}, n \in \mathbb{N}$. Clearly,

$$
\begin{aligned}
\lim _{n}\left\||x|+\lambda W\left(f_{n}\right)\right\|_{E(\mathcal{M}, \tau)} & =\lim _{n}\left\|W(\mu(x))+\lambda W\left(f_{n}\right)\right\|_{E(\mathcal{M}, \tau)} \\
& =\lim _{n}\left\|\mu(x)+\lambda f_{n}\right\|_{E}=1 .
\end{aligned}
$$


Let $x=u|x|$ be a polar decomposition of $x$. Since for $\lambda= \pm 1$,

$$
\begin{aligned}
\varlimsup_{n}\left\|x+\lambda u W\left(f_{n}\right)\right\|_{E(\mathcal{M}, \tau)} & =\varlimsup_{n}\left\|u|x|+\lambda u W\left(f_{n}\right)\right\|_{E(\mathcal{M}, \tau)} \\
& \leq \lim _{n}\left\||x|+\lambda W\left(f_{n}\right)\right\|_{E(\mathcal{M}, \tau)}=1
\end{aligned}
$$

and $\|x\|_{E(\mathcal{M}, \tau)}=1$, by Lemma 1.5 we have

$$
\lim _{n}\left\|x+\lambda u W\left(f_{n}\right)\right\|_{E(\mathcal{M}, \tau)}=1, \quad \lambda= \pm 1 .
$$

Using the assumption that $x$ is a strongly extreme point, we get the convergence $\left\|u W\left(f_{n}\right)\right\|_{E(\mathcal{M}, \tau)} \rightarrow 0$. Recall that $u^{*} u=s(x)$. Hence, also $\left\|s(x) W\left(f_{n}\right)\right\|_{E(\mathcal{M}, \tau)} \rightarrow 0$. But the image of the isomorphism $W$ is contained in $s(x) S(\mathcal{M}, \tau) s(x)$, where the unit element is $s(x)$. Therefore $s(x) W\left(f_{n}\right)=$ $W\left(f_{n}\right)$ and consequently

$$
\left\|f_{n}\right\|_{E}=\left\|\mu\left(f_{n}\right)\right\|_{E}=\left\|\mu\left(W\left(f_{n}\right)\right)\right\|_{E}=\left\|W\left(f_{n}\right)\right\|_{E(\mathcal{M}, \tau)} \rightarrow 0 .
$$

This concludes the proof in the case when $\tau(s(x))=\infty$.

Suppose now that $\tau(s(x))<\infty$. Thus $\mu_{\infty}(x)=0$. Let $x=u|x|$ be a polar decomposition of $x$. Since $s(x)=u^{*} u$ and $s\left(x^{*}\right)=u u^{*}$, we have $s(x) \sim s\left(x^{*}\right)$ and $\tau\left(s\left(x^{*}\right)\right)=\tau(s(x))<\infty$. Hence $s(x)$ and $s\left(x^{*}\right)$ are finite, equivalent projections in $\mathcal{M}$ and by [34, Chapter 5, Proposition 1.38], $n(x) \sim n\left(x^{*}\right)$. Therefore by Lemma 2.6 there exists an isometry $w$ such that $x=w|x|$.

Let $\left\|\mu(x)+\lambda f_{n}\right\|_{E} \rightarrow 1$ for $\lambda= \pm 1$, where $f_{n} \in B_{E}, n \in \mathbb{N}$. Proposition 1.2, applied to the operator $|x|$, implies the existence of a $*$-isomorphism $V$ from $S([0, \alpha), m)$ into $S(\mathcal{M}, \tau)$ such that $V(\mu(x))=|x|$ and $\mu(V(f))=$ $\mu(f)$ for all $f \in S([0, \alpha), m)$. Note that

$$
\begin{aligned}
\lim _{n}\left\||x| \pm V\left(f_{n}\right)\right\|_{E(\mathcal{M}, \tau)} & =\lim _{n}\left\|V(\mu(x)) \pm V\left(f_{n}\right)\right\|_{E(\mathcal{M}, \tau)} \\
& =\lim _{n}\left\|\mu(x) \pm f_{n}\right\|_{E}=1 .
\end{aligned}
$$

Since $w$ is an isometry, for $\lambda= \pm 1$ we have

$$
\begin{aligned}
\lim _{n}\left\|x+\lambda w V\left(f_{n}\right)\right\|_{E(\mathcal{M}, \tau)} & =\lim _{n}\left\|w|x|+\lambda w V\left(f_{n}\right)\right\|_{E(\mathcal{M}, \tau)} \\
& =\lim _{n}\left\||x|+\lambda V\left(f_{n}\right)\right\|_{E(\mathcal{M}, \tau)}=1 .
\end{aligned}
$$

Now as $x$ is a MLUR point of $B_{E(\mathcal{M}, \tau)}$, we get $\left\|w V\left(f_{n}\right)\right\|_{E(\mathcal{M}, \tau)} \rightarrow 0$. Hence

$$
\lim _{n}\left\|f_{n}\right\|_{E}=\lim _{n}\left\|V\left(f_{n}\right)\right\|_{E(\mathcal{M}, \tau)}=\lim _{n}\left\|w V\left(f_{n}\right)\right\|_{E(\mathcal{M}, \tau)}=0,
$$

which proves that $\mu(x)$ is a MLUR point of $B_{E}$, and ends the proof.

The next corollary combines the results of Theorems 2.5 and 2.7. It follows immediately from the well known fact that any MLUR space $E$ is order continuous [29]. 
COROLlary 2.8. Let $E$ be a fully symmetric function space on $[0, \alpha)$, $\alpha=\tau(\mathbf{1})$, and $\mathcal{M}$ be a von Neumann algebra with a faithful, normal, $\sigma$-finite trace $\tau$.

(1) Let $x$ be an order continuous element of $E(\mathcal{M}, \tau)$. Then $\mu(x)$ is a $M L U R$ point of $B_{E_{0}}$ if and only if $x$ is a $M L U R$ point of $B_{E_{0}(\mathcal{M}, \tau)}$.

(2) $E$ is a $M L U R$ space if and only if $E(\mathcal{M}, \tau)$ is a MLUR space.

Before proving the next theorem, we shall need a version of Lemma 2.1 for a symmetric sequence space $E$ and the unitary matrix space $C_{E}$. The proof of the atomic variant of Lemma 2.1 can be conducted in the same way as for symmetric function spaces, replacing singular value functions with sequences of singular numbers.

TheOREM 2.9. Let $E \subset c_{0}$ be a fully symmetric sequence space. Then $C_{E}$ is a MLUR space if and only if $E$ is a MLUR space.

Proof. Since $E$ is isometrically embedded in $C_{E}$ [2, Proposition 1.1], if $C_{E}$ is a MLUR space then so is $E$. As explained in the preliminary section, $C_{E}=G(B(H), \operatorname{tr})$ and $\|x\|_{C_{E}}=\|x\|_{G(B(H), \operatorname{tr})}$ for any compact operator $x$. Therefore proceeding as in the proof of Theorem 2.5. we can show that if $E$ is a MLUR space then so is $C_{E}$. Indeed, let $s(x)=\left\{s_{n}(x)\right\}_{n=1}^{\infty}$ be a MLUR point of $B_{E}$, and suppose that $\left\|2 x-y_{n}-z_{n}\right\|_{C_{E}} \rightarrow 0$, where $\left\{y_{n}\right\},\left\{z_{n}\right\} \subset B_{C_{E}}$. By Lemma 2.1 for the symmetric sequence space $E$,

$$
\begin{aligned}
& \left\|s(x)-s\left(y_{n}\right)\right\|_{E} \rightarrow 0, \quad\left\|s(x)-s\left(\left(y_{n}+x\right) / 2\right)\right\|_{E} \rightarrow 0 \\
& \left\|s(x)-s\left(z_{n}\right)\right\|_{E} \rightarrow 0, \quad\left\|s(x)-s\left(\left(z_{n}+x\right) / 2\right)\right\|_{E} \rightarrow 0 .
\end{aligned}
$$

Then, since $\mu(x)=\sum_{i=1}^{\infty} s_{i}(x) \chi_{[i-1, i)}$ and $\mu\left(y_{n}\right)=\sum_{i=1}^{\infty} s_{i}\left(y_{n}\right) \chi_{[i-1, i)}$ for all $n \in \mathbb{N}$, we have

$$
\left\|\mu(x)-\mu\left(y_{n}\right)\right\|_{G}=\left\|\sum_{i=1}^{\infty}\left(s_{i}(x)-s_{i}\left(y_{n}\right)\right) \chi_{[i-1, i)}\right\|_{G}=\left\|s(x)-s\left(y_{n}\right)\right\|_{E} \rightarrow 0 .
$$

Similarly, one can show that $\left\|\mu(x)-\mu\left(\left(y_{n}+x\right) / 2\right)\right\|_{G} \rightarrow 0,\left\|\mu(x)-\mu\left(z_{n}\right)\right\|_{G}$ $\rightarrow 0$ and $\left\|\mu(x)-\mu\left(\left(z_{n}+x\right) / 2\right)\right\|_{G} \rightarrow 0$. Now, by Lemma 2.2 it follows that $y_{n} \stackrel{\operatorname{tr}}{\longrightarrow} x$ and $z_{n} \stackrel{\operatorname{tr}}{\rightarrow} x$. Consequently, by Proposition 2.4 applied to the symmetric space $G(B(H)$, tr) it follows that

$\left\|x-y_{n}\right\|_{C_{E}}=\left\|x-y_{n}\right\|_{G(B(H), \operatorname{tr})} \rightarrow 0$ and $\left\|x-z_{n}\right\|_{C_{E}}=\left\|x-z_{n}\right\|_{G(B(H), \operatorname{tr})} \rightarrow 0$, proving that $x$ is a MLUR point of $B_{C_{E}}$. Therefore if $E$ is a MLUR space then so is $C_{E}$.

3. Complex extreme points and complex rotundity. The main result of this section, Theorem 3.11. gives a criterion for an operator $x$ to be a complex extreme point of $B_{E(\mathcal{M}, \tau)}$. This criterion is analogous to the characterization of extreme points obtained in [5, 18]. We will need several 
auxiliary results. The first two lemmas describe elementary characteristics of extreme points.

Lemma 3.1. Let $x, y \in B_{E(\mathcal{M}, \tau)}$ and suppose that $\mu_{t}(x) \leq \mu_{t}(y)$ for all $t \in[0, \infty)$. If there exists $t_{0}>0$ such that $\mu_{t_{0}}(x)<\mu_{t_{0}}(y)$ then $\mu(x)$ is not $a \mathbb{C}$-extreme point of $B_{E}$.

Proof. By the assumption $\mu(x), \mu(y) \in B_{E}$ and for all $t>0, \mu_{t}(x) \leq$ $\mu_{t}(y)$. Suppose that $\mu(x)$ is $\mathbb{C}$-extreme. Then by Theorem $1.4(1), \mu(x)$ is an UM point in its real part $E_{r}$. If $\mu_{t_{0}}(x)<\mu_{t_{0}}(y)$ for some $t_{0}>0$, then by the right continuity of the singular value function, there exists a set $A$ of positive measure such that $\mu_{t}(x)<\mu_{t}(y)$ for every $t \in A$. By the upper monotonicity of $\mu(x)$ we get

$$
1=\|\mu(x)\|_{E}<\|\mu(y)\|_{E},
$$

contradicting the fact that $\mu(y) \in B_{E}$.

Lemma 3.2. Let $x \in S(\mathcal{M}, \tau)$ be a self-adjoint operator. Then $x \in \mathbb{C}$ $\operatorname{ext}\left(B_{E_{h}(\mathcal{M}, \tau)}\right)$ if and only if $x \in \mathbb{C}-\operatorname{ext}\left(B_{E(\mathcal{M}, \tau)}\right)$.

Proof. It is enough to show one implication. Suppose that $x \in S_{E(\mathcal{M}, \tau)}$, $x=x^{*}$ and $x \in \mathbb{C}-\operatorname{ext}\left(() B_{E_{h}(\mathcal{M}, \tau)}\right)$. Let $x+\lambda y \in B_{E(\mathcal{M}, \tau)}, \lambda= \pm 1, \pm i$, where $y \in B_{E(\mathcal{M}, \tau)}$. Setting $y_{1}=\left(y^{*}+y\right) / 2$ and $y_{2}=\left(y-y^{*}\right) /(2 i)$, we get $y=y_{1}+i y_{2}$, where both $y_{1}$ and $y_{2}$ are self-adjoint. Note that for all $\lambda= \pm 1, \pm i$,

$$
\begin{aligned}
\left\|x+\lambda y_{1}\right\|_{E(\mathcal{M}, \tau)} & =\left\|x+\lambda\left(y+y^{*}\right) / 2\right\|_{E(\mathcal{M}, \tau)} \\
& \leq\|x+\lambda y\|_{E(\mathcal{M}, \tau)} / 2+\left\|x+\lambda y^{*}\right\|_{E(\mathcal{M}, \tau)} / 2 \\
& =\|x+\lambda y\|_{E(\mathcal{M}, \tau)} / 2+\|x+\bar{\lambda} y\|_{E(\mathcal{M}, \tau)} / 2 \leq 1
\end{aligned}
$$

and

$$
\begin{aligned}
\left\|x+\lambda y_{2}\right\|_{E(\mathcal{M}, \tau)} & =\left\|x+\lambda\left(y-y^{*}\right) /(2 i)\right\|_{E(\mathcal{M}, \tau)} \\
& \leq\|x-\lambda i y\|_{E(\mathcal{M}, \tau)} / 2+\left\|x+\lambda i y^{*}\right\|_{E(\mathcal{M}, \tau)} / 2 \\
& =\|x-\lambda i y\|_{E(\mathcal{M}, \tau)} / 2+\|x-\bar{\lambda} i y\|_{E(\mathcal{M}, \tau)} / 2 \leq 1 .
\end{aligned}
$$

As $x$ is a $\mathbb{C}$-extreme point, it follows that $y_{1}=y_{2}=0$ and so $y=0$.

Lemma 3.3. For any $x \in S(\mathcal{M}, \tau), n(x) S(\mathcal{M}, \tau) n\left(x^{*}\right)=0$ whenever $n(x) \mathcal{M} n\left(x^{*}\right)=0$.

Proof. Suppose that $n(x) \mathcal{M} n\left(x^{*}\right)=0$ and let $y \in S(\mathcal{M}, \tau)$. Recall that if $a$ is a closed linear operator with the domain $\mathcal{D}(a)=H$, then by the Closed Graph Theorem, $a \in B(H)$. Furthermore, if $a$ is a bounded linear operator affiliated with $\mathcal{M}$, that is, $b a=a b$ for all $b \in \mathcal{M}^{\prime}$, then $a \in\left(\mathcal{M}^{\prime}\right)^{\prime}=\mathcal{M}$. Since $e^{|y|}[0, n](H) \subset \mathcal{D}(|y|)=\mathcal{D}(y)$, we have $y e^{|y|}[0, n] \in B(H)$ and by the $\tau$-measurability of $y, y e^{|y|}[0, n]$ is affiliated with $\mathcal{M}$. Hence for all $n \in \mathbb{N}$, 
$y e^{|y|}[0, n] \in \mathcal{M}$ and by assumption $n(x) y e^{|y|}[0, n] n\left(x^{*}\right)=0$. We will now show that

$$
n(x) y e^{|y|}[0, n] n\left(x^{*}\right) \stackrel{\tau}{\rightarrow} n(x) y n\left(x^{*}\right) \quad \text { as } n \rightarrow \infty .
$$

By the $\tau$-measurability of $|y|$ there exists $n_{1} \in \mathbb{N}$ for which $\tau\left(e^{|y|}\left(n_{1}, \infty\right)\right)$ $<\infty$. Since

$$
e^{|y|}\left(n_{1}, \infty\right)-e^{|y|}(n, \infty) \uparrow e^{|y|}\left(n_{1}, \infty\right),
$$

by the normality of trace $\tau$ it follows that

$$
\tau\left(e^{|y|}\left(n_{1}, \infty\right)-e^{|y|}(n, \infty)\right) \rightarrow \tau\left(e^{|y|}\left(n_{1}, \infty\right)\right),
$$

where for $n \geq n_{1}$,

$$
\tau\left(e^{|y|}\left(n_{1}, \infty\right)-e^{|y|}(n, \infty)\right)=\tau\left(e^{|y|}\left(n_{1}, \infty\right)\right)-\tau\left(e^{|y|}(n, \infty)\right) .
$$

Thus $\tau\left(e^{|y|}(n, \infty)\right) \rightarrow 0$ as $n \rightarrow \infty$. Consequently, for all $t \in \mathbb{R}^{+}$,

$$
\begin{aligned}
\mu_{t}\left(n(x) y e^{|y|}[0, n] n\left(x^{*}\right)\right. & \left.-n(x) y n\left(x^{*}\right)\right)=\mu_{t}\left(n(x) y e^{|y|}(n, \infty) n\left(x^{*}\right)\right) \\
\leq & \mu_{t}\left(y e^{|y|}(n, \infty)\right)=\mu_{t}(y) \chi_{\left[0, \tau\left(e^{|y|}(n, \infty)\right)\right)}(t) \rightarrow 0 .
\end{aligned}
$$

It follows that $n(x) y e^{|y|}[0, n] n\left(x^{*}\right) \stackrel{\tau}{\rightarrow} n(x) y n\left(x^{*}\right)$ [19, Lemma 3.4], and since $n(x) y e^{|y|}[0, n] n\left(x^{*}\right)=0$ for all $n \in \mathbb{N}$, the claim follows.

Lemma 3.4. Let $x \in S(\mathcal{M}, \tau)$. Then $|x| \geq \mu_{\infty}(x) s(x)$ if and only if $\left|x^{*}\right| \geq \mu_{\infty}(x) s\left(x^{*}\right)$.

Proof. Suppose that $|x| \geq \mu_{\infty}(x) s(x)$. Let $x=u|x|$ be a polar decomposition of $x$. Then $u|x| u^{*}=\left|x^{*}\right|$ and $\left|x^{*}\right| \geq \mu_{\infty}(x) u s(x) u^{*}$. Indeed,

$$
\begin{aligned}
\left\langle\left|x^{*}\right| \xi, \xi\right\rangle & =\left\langle u|x| u^{*} \xi, \xi\right\rangle=\left\langle|x| u^{*} \xi, u^{*} \xi\right\rangle \geq \mu_{\infty}(x)\left\langle s(x) u^{*} \xi, u^{*} \xi\right\rangle \\
& =\mu_{\infty}(x)\left\langle u s(x) u^{*} \xi, \xi\right\rangle
\end{aligned}
$$

for any $\xi$ in the domain of $\left|x^{*}\right|$. Applying now the well known equalities $s(x)=u^{*} u$ and $s\left(x^{*}\right)=u u^{*}$, we get $u s(x) u^{*}=u u^{*} u u^{*}=s\left(x^{*}\right)$, and so $\left|x^{*}\right| \geq \mu_{\infty}(x) s\left(x^{*}\right)$.

Conversely, if $\left|x^{*}\right| \geq \mu_{\infty}(x) s\left(x^{*}\right)$, then by the above argument $|x|=$ $\left|\left(x^{*}\right)^{*}\right| \geq \mu_{\infty}(x) s(x)$.

We shall need the following results, in particular Corollary 3.6, to prove that $x$ is a complex extreme point whenever $\mu(x)$ is.

Lemma 3.5. Let $x \in S(\mathcal{M}, \tau)$ and $x \geq \mu_{\infty}(x) \mathbf{1}$. If $\mu(x) \in \mathbb{C}$-ext $\left(B_{E}\right)$ then $x \in \mathbb{C}-\operatorname{ext}\left(B_{E(\mathcal{M}, \tau)}\right)$.

Proof. Suppose that $\mu(x) \in \mathbb{C}$-ext $\left(B_{E}\right)$ and $x \pm y, x \pm i y$ belong to $B_{E(\mathcal{M}, \tau)}$ for some $y \in B_{E(\mathcal{M}, \tau)}$. In view of Lemma 3.2, we can assume that $y$ is a self-adjoint operator. Now by Proposition 1.1(4), for all $t>0$,

$$
\mu_{t}(x) \leq \mu_{t}(x+i y) \text {. }
$$


Since $\mu(x) \in \mathbb{C}$-ext $\left(B_{E}\right)$ and $\mu(x+i y) \in B_{E}$, by Lemma 3.1 it follows that for all $t>0$,

$$
\mu_{t}(x)=\mu_{t}(x+i y) .
$$

Then Proposition 1.1 (5) implies that $y=0$, and the claim follows.

Corollary 3.6. If $\mu(x) \in \mathbb{C}-\operatorname{ext}\left(B_{E}\right)$ and $|x| \geq \mu_{\infty}(x) s(x)$, then $|x|+\mu_{\infty}(x) n(x) \in \mathbb{C}-\operatorname{ext}\left(B_{E(\mathcal{M}, \tau)}\right)$. Consequently, if $\mu(x) \in \mathbb{C}-\operatorname{ext}\left(B_{E}\right)$ and $\mu_{\infty}(x)=0$, then $|x| \in \mathbb{C}-\operatorname{ext}\left(B_{E(\mathcal{M}, \tau)}\right)$.

Proof. This follows immediately from Lemma 3.5, since $|x| \geq \mu_{\infty}(x) s(x)$ implies that $|x|+\mu_{\infty}(x) n(x) \geq \mu_{\infty}(x) \mathbf{1}$, and by Proposition 1.1(1), we have $\mu\left(|x|+\mu_{\infty}(x) n(x)\right)=\mu(x)$.

We are now ready for our first main claim in this section.

Theorem 3.7. An element $x \in S_{E(\mathcal{M}, \tau)}$ is a $\mathbb{C}$-extreme point of $B_{E(\mathcal{M}, \tau)}$ whenever $\mu(x)$ is a $\mathbb{C}$-extreme point of $B_{E}$ and one of the following conditions holds:

(i) $\mu_{\infty}(x)=0$,

(ii) $n(x) \mathcal{M} n\left(x^{*}\right)=0$ and $|x| \geq \mu_{\infty}(x) s(x)$.

Proof. Suppose that $\mu(x)$ is a $\mathbb{C}$-extreme point and $x \pm y, x \pm i y$ belong to $B_{E(\mathcal{M}, \tau)}$ for some $y \in B_{E(\mathcal{M}, \tau)}$. Let $x=u|x|$ and $x^{*}=u^{*}\left|x^{*}\right|$ be polar decompositions of $x$ and $x^{*}$, respectively. Since Ker $u=\operatorname{Ker} x$ and Ker $u^{*}=$ Ker $x^{*}$, we have $u n(x)=u^{*} n\left(x^{*}\right)=0$. Hence $x=u\left(|x|+\mu_{\infty}(x) n(x)\right)$ and $x^{*}=u^{*}\left(\left|x^{*}\right|+\mu_{\infty}(x) n\left(x^{*}\right)\right)$. Thus $|x|+\mu_{\infty}(x) n(x)=u^{*} x$ and $\left|x^{*}\right|+$ $\mu_{\infty}(x) n\left(x^{*}\right)=u x^{*}$, and so

$$
|x|+\mu_{\infty}(x) n(x)+\lambda u^{*} y,\left|x^{*}\right|+\mu_{\infty}(x) n\left(x^{*}\right)+\lambda u y^{*} \in B_{E(\mathcal{M}, \tau)}
$$

for all $\lambda= \pm 1, \pm i$.

In view of the assumption (i) or (ii) and Lemma 3.4. $|x| \geq \mu_{\infty}(x) s(x)$ and $\left|x^{*}\right| \geq \mu_{\infty}(x) s\left(x^{*}\right)$. Since $\mu(x)=\mu\left(x^{*}\right)$ is a $\mathbb{C}$-extreme point, Corollary 3.6 implies that $|x|+\mu_{\infty}(x) n(x)$ and $\left|x^{*}\right|+\mu_{\infty}(x) n\left(x^{*}\right)$ are complex extreme points of $B_{E(\mathcal{M}, \tau)}$. Therefore $u^{*} y=u y^{*}=0$. Hence $s\left(x^{*}\right) y=u u^{*} y=0$ and $y s(x)=y u^{*} u=0$, since $\left(y u^{*} u\right)^{*}=u^{*} u y^{*}=0$. It follows that $y=$ $\left(s\left(x^{*}\right)+n\left(x^{*}\right)\right) y(s(x)+n(x))=n\left(x^{*}\right) y n(x)$.

If (ii) is satisfied, then by Lemma 3.3, $n\left(x^{*}\right) S(\mathcal{M}, \tau) n(x)=0$ and consequently $y=n\left(x^{*}\right) y n(x)=0$.

Consider now the case when (i) holds true, that is, $\mu_{\infty}(x)=0$. Then

$$
\begin{aligned}
|x+\lambda y|^{2} & =\left|x+\lambda n\left(x^{*}\right) y n(x)\right|^{2}=\left(x+\lambda n\left(x^{*}\right) y n(x)\right)^{*}\left(x+\lambda n\left(x^{*}\right) y n(x)\right) \\
& =\left(x^{*}+\bar{\lambda} n(x) y^{*} n\left(x^{*}\right)\right)\left(x+\lambda n\left(x^{*}\right) y n(x)\right) \\
& =x^{*} x+\lambda x^{*} n\left(x^{*}\right) y n(x)+\bar{\lambda} n(x) y^{*} n\left(x^{*}\right) x+n(x) y^{*} n\left(x^{*}\right) n\left(x^{*}\right) y n(x) \\
& =|x|^{2}+\left|n\left(x^{*}\right) y n(x)\right|^{2} .
\end{aligned}
$$


Also

$$
\begin{aligned}
(|x|+|y|)^{2} & =\left(|x|+\left|n\left(x^{*}\right) y n(x)\right|\right)^{2} \\
& =|x|^{2}+|x|\left|n\left(x^{*}\right) y n(x)\right|+\left|n\left(x^{*}\right) y n(x)\right||x|+\left|n\left(x^{*}\right) y n(x)\right|^{2} .
\end{aligned}
$$

Let $n\left(x^{*}\right) y n(x)=v\left|n\left(x^{*}\right) y n(x)\right|$ be a polar decomposition of $n\left(x^{*}\right) y n(x)$. Then $\left|n\left(x^{*}\right) y n(x)\right|=v^{*} n\left(x^{*}\right) y n(x)=n(x) y^{*} n\left(x^{*}\right) v$, and so $|x|\left|n\left(x^{*}\right) y n(x)\right|$ $=u^{*} x n(x) y^{*} n\left(x^{*}\right) v=0$. Also $\left|n\left(x^{*}\right) y n(x)\right||x|=0$. Hence

$$
|x+\lambda y|=|x|+|y| \quad \text { for } \lambda= \pm 1, \pm i,
$$

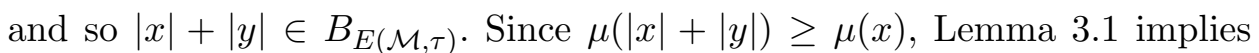
that $\mu(|x|+|y|)=\mu(x)$. By Proposition 1.1(3), if $|y| \neq 0$ then $\mu_{s}(|x|+|y|)>$ $\mu_{s}(|x|)$ for some $s>0$. Thus $|y|=0$, and consequently $y=0$. This concludes the proof in the case when $\mu_{\infty}(x)=0$.

To show the converse statement we will need the next two lemmas.

Lemma 3.8. If $x$ is a $\mathbb{C}$-extreme point of $B_{E(\mathcal{M}, \tau)}$ then $|x| \geq \mu_{\infty}(x) s(x)$.

Proof. Suppose that $\mu_{\infty}(x)>0$. Let $x=u|x|$ be a polar decomposition of $x$. Fix $0<\epsilon<1$ and consider the operators

$$
a_{ \pm}=|x| \pm \epsilon|x| e^{|x|}[0, \beta], \quad b_{ \pm}=|x| \pm i \epsilon|x| e^{|x|}[0, \beta],
$$

where $\beta=\frac{1}{1+\epsilon} \mu_{\infty}(x)$. Clearly,

$$
\begin{aligned}
& a_{-}=|x| e^{|x|}(\beta, \infty)+(1-\epsilon)|x| e^{|x|}[0, \beta], \\
& a_{+}=|x| e^{|x|}(\beta, \infty)+(1+\epsilon)|x| e^{|x|}[0, \beta] .
\end{aligned}
$$

Hence $0 \leq a_{-} \leq|x|$, and so $\mu\left(a_{-}\right) \leq \mu(x)$. Consider now the real-valued function $f(t)=(1+\epsilon) t \chi_{[0, \beta]}+t \chi_{(\beta, \infty)}, t \geq 0$. We have $a_{+}=f(|x|)$, and it follows that

$$
e^{a_{+}}(\lambda, \infty)=e^{|x|}\left(f^{-1}(\lambda, \infty)\right)= \begin{cases}e^{|x|}\left(\frac{1}{1+\epsilon} \lambda, \infty\right) & \text { if } \lambda<\mu_{\infty}(x), \\ e^{|x|}(\lambda, \infty) & \text { if } \lambda \geq \mu_{\infty}(x),\end{cases}
$$

for all $\lambda \geq 0$. Since $\mu_{\infty}(x)=\inf \left\{s \geq 0: \tau\left(e^{|x|}(s, \infty)\right)<\infty\right\}$, we have $\tau\left(e^{a_{+}}(\lambda, \infty)\right)=\infty$ for all $\lambda<\mu_{\infty}(x)$. Thus for any $t>0$,

$$
\begin{aligned}
\mu_{t}\left(a_{+}\right) & =\inf \left\{\lambda: \tau\left(e^{a_{+}}(\lambda, \infty)\right) \leq t\right\}=\inf \left\{\lambda \geq \mu_{\infty}(x): \tau\left(e^{|x|}(\lambda, \infty)\right) \leq t\right\} \\
& =\mu_{t}(x),
\end{aligned}
$$

which yields $\mu\left(a_{+}\right)=\mu(x)$. Now observe that

$$
\begin{aligned}
\left|b_{-}\right|^{2} & =\left|b_{+}\right|^{2}=b_{-}^{*} b_{-}=b_{+}^{*} b_{+}=|x|^{2}+\epsilon^{2}|x|^{2} e^{|x|}[0, \beta] \\
& \leq|x|^{2}+\epsilon^{2}|x|^{2} e^{|x|}[0, \beta]+2 \epsilon|x|^{2} e^{|x|}[0, \beta]=\left(|x|+\epsilon|x| e^{|x|}[0, \beta]\right)^{2}=a_{+}^{2} .
\end{aligned}
$$


Hence

$$
\mu^{2}\left(b_{-}\right)=\mu^{2}\left(b_{+}\right)=\mu\left(\left|b_{+}\right|^{2}\right)=\mu\left(\left|b_{-}\right|^{2}\right) \leq \mu^{2}\left(a_{+}\right)=\mu^{2}(x)
$$

and $\mu\left(b_{+}\right)=\mu\left(b_{-}\right) \leq \mu(x)$.

Thus $|x|+\lambda \epsilon|x| e^{|x|}[0, \beta] \in B_{E(\mathcal{M}, \tau)}$, and so $x+\lambda \epsilon u|x| e^{|x|}[0, \beta] \in B_{E(\mathcal{M}, \tau)}$ for all $\lambda= \pm 1, \pm i$. As $x$ is a $\mathbb{C}$-extreme point, $u|x| e^{|x|}[0, \beta]=0$. But $u^{*} u|x|=$ $|x|$ and therefore

$$
|x| e^{|x|}[0, \beta]=|x| e^{|x|}\left[0, \frac{1}{1+\epsilon} \mu_{\infty}(x)\right]=0 \quad \text { for every } 0<\epsilon<1 .
$$

Since $\epsilon$ can be arbitrarily small, it follows that $|x| e^{|x|}\left[0, \mu_{\infty}(x)\right)=0$. Hence $0=\int_{0}^{\mu_{\infty}(x)} \lambda d e^{|x|}(\lambda)$, which implies that $e^{|x|}\left(0, \mu_{\infty}(x)\right)=0$. Therefore $s(x)=$ $e^{|x|}(0, \infty)=e^{|x|}\left[\mu_{\infty}(x), \infty\right)$, and finally

$$
|x|=\int_{\left[\mu_{\infty}(x), \infty\right)} \lambda d e^{|x|}(\lambda) \geq \mu_{\infty}(x) e^{|x|}\left[\mu_{\infty}(x), \infty\right)=\mu_{\infty}(x) s(x) .
$$

Lemma 3.9. If $x$ is a $\mathbb{C}$-extreme point of $B_{E(\mathcal{M}, \tau)}$ then $n(x) \mathcal{M} n\left(x^{*}\right)=0$ or $\mu_{\infty}(x)=0$.

Proof. Assume for a contradiction that $n(x) \mathcal{M} n\left(x^{*}\right) \neq 0$ and $\mu_{\infty}(x)>0$, while $x$ is a complex extreme point. By [34, Chapter 5, Lemma 1.7] there exist nonzero projections $p, q \in \mathcal{P}(\mathcal{M})$ such that $p \leq n(x), q \leq n\left(x^{*}\right)$ and $p \sim q$, that is, there exists a partial isometry $v$ such that $p=v^{*} v$ and $q=v v^{*}$.

Let $x=u|x|$ be a polar decomposition of $x$. Set $w=u+v$. We claim that $w$ is a partial isometry and $x=w|x|$. Indeed, since Ker $u^{*}=\operatorname{Ker} x^{*}$ we have $u^{*} n\left(x^{*}\right)=0$ and $u^{*} q=0$. Now $0=u^{*} q u=u^{*} v v^{*} u=\left(v^{*} u\right)^{*}\left(v^{*} u\right)=\left|v^{*} u\right|^{2}$. Therefore $v^{*} u=0$ and $u^{*} v=0$. Hence

$$
\begin{aligned}
|w|^{2} & =|u+v|^{2}=\left(u^{*}+v^{*}\right)(u+v)=u^{*} u+u^{*} v+v^{*} u+v^{*} v \\
& =u^{*} u+v^{*} v=s(x)+p,
\end{aligned}
$$

and thus $w$ is a partial isometry, since $w^{*} w$ is a projection. Now, since $0=(|x| n(x) p)^{*}=(|x| p)^{*}=p|x|=v^{*} v|x|$ and $v^{*} v$ is a projection onto $\operatorname{Ker}^{\perp} v$, it follows that Range $|x| \subset \operatorname{Ker} v$, and therefore $v|x|=0$. Hence $x 1=u|x|=u|x|+v|x|=w|x|$.

Note that ||$x\left|+\lambda \mu_{\infty}(x) n(x)\right|=|x|+\mu_{\infty}(x) n(x)$ for all $\lambda= \pm 1, \pm i$, since $|x| n(x)=n(x)|x|=0$. Furthermore, by Proposition 1.1 (1), we have $\mu\left(|x|+\mu_{\infty}(x) n(x)\right)=\mu(x)$. Hence $|x|+\lambda \mu_{\infty}(x) n(x) \in B_{E(\mathcal{M}, \tau)}$ for all $\lambda= \pm 1, \pm i$. Moreover,

$$
\begin{aligned}
\mu\left(x+\lambda \mu_{\infty}(x) w n(x)\right) & =\mu\left(w|x|+\lambda \mu_{\infty}(x) w n(x)\right) \\
& \leq \mu\left(|x|+\lambda \mu_{\infty}(x) n(x)\right)=\mu(x),
\end{aligned}
$$


which implies that $x+\lambda \mu_{\infty}(x) w n(x) \in B_{E(\mathcal{M}, \tau)}$ for all $\lambda= \pm 1, \pm i$. The assumption that $x$ is a $\mathbb{C}$-extreme point yields $\mu_{\infty}(x) w n(x)=0$. But $\mu_{\infty}(x)>0$ and therefore $w n(x)=0$. Since $x=u|x|$, we know that $\operatorname{Ker} u=\operatorname{Ker} x$, and so $u n(x)=0$. Hence $0=w n(x)=(u+v) n(x)=v n(x)$. But then

$$
p=p n(x)=v^{*} \operatorname{vn}(x)=0,
$$

which contradicts the fact that $p \neq 0$.

Theorem 3.10. Suppose that the trace $\tau$ on $\mathcal{M}$ is $\sigma$-finite. If $x$ is a complex extreme point of $B_{E(\mathcal{M}, \tau)}$ then $\mu(x)$ is a complex extreme point of $B_{E}$ and either

(i) $\mu_{\infty}(x)=0$, or

(ii) $n(x) \mathcal{M} n\left(x^{*}\right)=0$ and $|x| \geq \mu_{\infty}(x) s(x)$.

Proof. Suppose that $x$ is a $\mathbb{C}$-extreme point of the unit ball in $E(\mathcal{M}, \tau)$. Since $\tilde{\pi}: x \mapsto \mathbb{1} \otimes x$ is a $*$-isometry from $E(\mathcal{M}, \tau)$ onto $E(\mathbb{C} \mathbb{1} \otimes \mathcal{M}, \kappa)$, clearly $\mathbb{1} \otimes x$ is a $\mathbb{C}$-extreme point in $E(\mathbb{C} \mathbb{1} \otimes \mathcal{M}, \kappa)$, where $\mathbb{C} \mathbb{1} \otimes \mathcal{M}$ is a nonatomic von Neumann algebra. Moreover, $\tilde{\pi}$ preserves the singular value function, and therefore we can assume that the von Neumann algebra $\mathcal{M}$ is nonatomic.

Consider first the case $\tau(s(x))=\infty$. By Lemma 3.8, $|x| \geq \mu_{\infty}(x) s(x)$. Also $\mu\left(|x|-\mu_{\infty}(x) s(x)\right)=\mu(x)-\mu_{\infty}(x)$ by Proposition 1.1 (2), and consequently $\mu_{\infty}\left(|x|-\mu_{\infty}(x) s(x)\right)=0$. Clearly,

$$
s(x)\left(|x|-\mu_{\infty}(x) s(x)\right)=\left(|x|-\mu_{\infty}(x) s(x)\right) s(x)=|x|-\mu_{\infty}(x) s(x) .
$$

Proposition 1.3 applied to the operator $|x|-\mu_{\infty}(x) s(x)$ and $p=s(x)$ yields a $*$-isomorphism $W$ from $S([0, \infty), m)$ into $s(x) S(\mathcal{M}, \tau) s(x)$ such that

$$
W\left(\mu\left(|x|-\mu_{\infty}(x) s(x)\right)\right)=|x|-\mu_{\infty}(x) s(x) \quad \text { and } \quad \mu(W(f))=\mu(f)
$$

for all $f \in S([0, \infty), m)$. Since $W(1)=s(x)$,

$$
\begin{aligned}
|x|-\mu_{\infty}(x) s(x) & =W\left(\mu\left(|x|-\mu_{\infty}(x) s(x)\right)\right)=W\left(\mu(x)-\mu_{\infty}(x)\right) \\
& =W(\mu(x))-\mu_{\infty}(x) W(1)=W(\mu(x))-\mu_{\infty}(x) s(x),
\end{aligned}
$$

and consequently $W(\mu(x))=|x|$.

Let now $\mu(x)+\lambda f \in B_{E}$ for all $\lambda= \pm 1, \pm i$, where $f \in B_{E}$. Since $W$ is an isometry, $\||x|+\lambda W(f)\|_{E(\mathcal{M}, \tau)}=\|W(\mu(x))+\lambda W(f)\|_{E(\mathcal{M}, \tau)} \leq 1$ for $\lambda= \pm 1, \pm i$. Let $x=u|x|$ be a polar decomposition of $x$. Clearly,

$$
x+\lambda u W(f)=u|x|+\lambda u W(f) \in B_{E(\mathcal{M}, \tau)}, \quad \lambda= \pm 1, \pm i .
$$

Since $x$ is a $\mathbb{C}$-extreme point, $u W(f)=0$. Recall that $u^{*} u=s(x)$. Hence $s(x) W(f)=0$. Note also that $W(f) \in s(x) S(\mathcal{M}, \tau) s(x)$. Therefore $W(f)=$ $s(x) W(f)=0$, and consequently $f=0$.

Consider now the case when $\tau(s(x))<\infty$, and hence $\mu_{\infty}(x)=0$. By Proposition 1.2 applied to $|x|$, there exists a $*$-isomorphism $V$ from 
$S([0, \alpha), m)$ into $S(\mathcal{M}, \tau)$ such that $V(\mu(x))=|x|$ and $\mu(V(f))=\mu(f)$ for all $f \in S([0, \alpha), m)$. Furthermore, since $s(x) \sim s\left(x^{*}\right)$, we have $\tau\left(s\left(x^{*}\right)\right)=$ $\tau(s(x))<\infty$ and it follows from [34, Chapter 5, Proposition 1.38] that $n(x) \sim n\left(x^{*}\right)$. Hence by Lemma 2.6, there exists an isometry $w$ such that $x=w|x|$.

Let $\mu(x)+\lambda f \in B_{E}, \lambda= \pm 1, \pm i$ and $f \in B_{E}$. Then

$$
\begin{aligned}
\|x+\lambda w V(f)\|_{E(\mathcal{M}, \tau)} & =\|w|x|+\lambda w V(f)\|_{E(\mathcal{M}, \tau)}=\||x|+\lambda V(f)\|_{E(\mathcal{M}, \tau)} \\
& =\|V(\mu(x))+\lambda V(f)\|_{E(\mathcal{M}, \tau)}=\|\mu(x)+\lambda f\|_{E} \leq 1 .
\end{aligned}
$$

Now by the assumption that $x$ is a $\mathbb{C}$-extreme point, $w V(f)=0$. Since both $w$ and $V$ are injective, $f=0$.

We finish this section with a complete characterization of $\mathbb{C}$-extreme points in $B_{E(\mathcal{M}, \tau)}$. The first result is an immediate consequence of Theorems 3.7 and 3.10 ,

TheOREM 3.11. Let $E$ be a symmetric space on $[0, \alpha), \alpha=\tau(\mathbf{1})$, and $\mathcal{M}$ be a semifinite von Neumann algebra with a faithful, normal, $\sigma$-finite trace $\tau$. An operator $x$ is a complex extreme point of $B_{E(\mathcal{M}, \tau)}$ if and only if $\mu(x)$ is a complex extreme point of $B_{E}$ and one of the following, not mutually exclusive, conditions holds:

(i) $\mu_{\infty}(x)=0$,

(ii) $n(x) \mathcal{M} n\left(x^{*}\right)=0$ and $|x| \geq \mu_{\infty}(x) s(x)$.

Since $\mu_{\infty}(x)=0$ for every $x \in E_{0}$, we immediately obtain the following corollary.

COROllary 3.12. Let $E$ be a symmetric space on $[0, \alpha), \alpha=\tau(\mathbf{1})$ and $\mathcal{M}$ be a semifinite von Neumann algebra with a faithful, normal, $\sigma$-finite trace $\tau$. Then $E_{0}$ is complex rotund if and only if $E_{0}(\mathcal{M}, \tau)$ is complex rotund.

TheOREM 3.13. Let $E \subset c_{0}$ be a symmetric sequence space. Then $C_{E}$ is complex rotund if and only if $E$ is complex rotund.

Proof. Since $E$ is isometrically embedded in $C_{E}$ [2], the claim that $E$ is a complex rotund space if $C_{E}$ has this property is immediate. As $C_{E}=$ $G(B(H), \operatorname{tr})$ and $\|x\|_{C_{E}}=\|x\|_{G(B(H), \text { tr })}$ for any compact operator $x$, the proof of the converse implication is analogous to the proof of Theorem 3.7 .

\section{Complex local uniform rotund points and complex local uni-} form rotundity. In this section we study the relations between the complex local uniform rotundity of the symmetric function space $E$ and the complex local uniform rotundity of the corresponding symmetric space $E(\mathcal{M}, \tau)$ of measurable operators. 
THEOREM 4.1. Let $E$ be strongly symmetric and $x$ be an order continuous element of $E(\mathcal{M}, \tau)$. If $\mu(x)$ is a $\mathbb{C}$-LUR point of $B_{E_{0}}$ then $x$ is a $\mathbb{C}$ - $L U R$ point of $B_{E_{0}(\mathcal{M}, \tau)}$.

Proof. Let $x \in S_{E_{0}(\mathcal{M}, \tau)}$ and suppose that $\mu(x)$ is a $\mathbb{C}$-LUR point of $B_{E_{0}}$.

CASE 1. Let $x \geq 0$ and $\left\{y_{n}\right\} \subset B_{E_{h}(\mathcal{M}, \tau)},\left\{y_{n}\right\} \subset E_{0}(\mathcal{M}, \tau)$, be such that $\left\|x+i y_{n}\right\|_{E(\mathcal{M}, \tau)} \rightarrow 1$. By Proposition 1.1 $(4), \mu_{t}\left(x+i y_{n}\right) \geq \mu_{t}(x)$ for all $t>0$. Since $\mu(x)$ is an ULUM point in $E_{0}$ by Theorem 1.4 (2), we have

$$
\left\|\mu(x)-\mu\left(x+i y_{n}\right)\right\|_{E} \rightarrow 0 .
$$

Also $\mu_{t}\left(x+i y_{n} / 2\right) \geq \mu_{t}(x)$ for all $t>0$ and $\left\|x+i y_{n} / 2\right\|_{E(\mathcal{M}, \tau)} \rightarrow 1$. The latter follows from the inequality $1 \leq\left\|x+i y_{n} / 2\right\|_{E(\mathcal{M}, \tau)} \leq\left\|x+i y_{n}\right\|_{E(\mathcal{M}, \tau)} / 2+$ $\|x\|_{E(\mathcal{M}, \tau)} / 2 \rightarrow 1$ as $n \rightarrow \infty$. Again, using the fact that $\mu(x)$ is an ULUM point we conclude that

$$
\left\|\mu(x)-\mu\left(x+i y_{n} / 2\right)\right\|_{E} \rightarrow 0 .
$$

Denote $a_{n}=x+i y_{n}$. From (4.2) it follows that $\left\|\mu(x)-\mu\left(\left(x+a_{n}\right) / 2\right)\right\|_{E}$ $\rightarrow 0$, and from 4.1 that $\left\|\mu(x)-\mu\left(a_{n}\right)\right\|_{E} \rightarrow 0$. Applying now Lemma 2.2 we get $a_{n} \stackrel{\tau}{\rightarrow} x$. Finally, Proposition 2.4 implies that $\left\|y_{n}\right\|_{E(\mathcal{M}, \tau)}=$ $\left\|x-a_{n}\right\|_{E(\mathcal{M}, \tau)} \rightarrow 0$.

CASE 2. Let $\left\|x+\lambda y_{n}\right\|_{E(\mathcal{M}, \tau)} \rightarrow 1$ for $\lambda= \pm 1, \pm i$, where $x \geq 0$ and $\left\{y_{n}\right\} \subset B_{E_{0}(\mathcal{M}, \tau)}$. Recall that for any $n \in \mathbb{N}, \operatorname{Re} y_{n}=\left(y_{n}+y_{n}^{*}\right) / 2, \operatorname{Im} y_{n}=$ $\left(y_{n}-y_{n}^{*}\right) /(2 i)$ are self-adjoint operators and $y_{n}=\operatorname{Re} y_{n}+i \operatorname{Im} y_{n}$. Note that by Proposition $1.1(4), \mu\left(x+i\left(y_{n}+y_{n}^{*}\right) / 2\right) \geq \mu(x)$, and thus

$$
\begin{aligned}
1 \leq\left\|x+i \operatorname{Re} y_{n}\right\|_{E(\mathcal{M}, \tau)} & \leq \frac{1}{2}\left\|x+i y_{n}\right\|_{E(\mathcal{M}, \tau)}+\frac{1}{2}\left\|x+i y_{n}^{*}\right\|_{E(\mathcal{M}, \tau)} \\
& =\frac{1}{2}\left\|x+i y_{n}\right\|_{E(\mathcal{M}, \tau)}+\frac{1}{2}\left\|x-i y_{n}\right\|_{E(\mathcal{M}, \tau)} .
\end{aligned}
$$

Thus $\left\|x+i \operatorname{Re} y_{n}\right\|_{E(\mathcal{M}, \tau)} \rightarrow 1$, and by Case $1,\left\|\operatorname{Re} y_{n}\right\|_{E(\mathcal{M}, \tau)} \rightarrow 0$. Similarly, the inequality

$$
\begin{aligned}
1 \leq\left\|x+i \operatorname{Im} y_{n}\right\|_{E(\mathcal{M}, \tau)} & \leq \frac{1}{2}\left\|x+y_{n}\right\|_{E(\mathcal{M}, \tau)}+\frac{1}{2}\left\|x-y_{n}^{*}\right\|_{E(\mathcal{M}, \tau)} \\
& =\frac{1}{2}\left\|x+y_{n}\right\|_{E(\mathcal{M}, \tau)}+\frac{1}{2}\left\|x-y_{n}\right\|_{E(\mathcal{M}, \tau)}
\end{aligned}
$$

implies that $\left\|x+i \operatorname{Im} y_{n}\right\|_{E(\mathcal{M}, \tau)} \rightarrow 1$, and consequently $\left\|\operatorname{Im} y_{n}\right\|_{E(\mathcal{M}, \tau)} \rightarrow 0$. Therefore $\left\|y_{n}\right\|_{E(\mathcal{M}, \tau)} \rightarrow 0$.

CASE 3. Suppose now that $x$ is an arbitrary element of $S_{E_{0}(\mathcal{M}, \tau)},\left\{y_{n}\right\} \subset$ $B_{E_{0}(\mathcal{M}, \tau)}$ and $\left\|x+\lambda y_{n}\right\|_{E(\mathcal{M}, \tau)} \rightarrow 1$ for all $\lambda= \pm 1, \pm i$. Let $x=u|x|$ be a polar decomposition of $x$. Then for all $\lambda= \pm 1, \pm i,\left\||x|+\lambda u^{*} y_{n}\right\|_{E(\mathcal{M}, \tau)}=$ $\left\|u^{*} x+\lambda u^{*} y_{n}\right\|_{E(\mathcal{M}, \tau)} \leq\left\|x+\lambda y_{n}\right\|_{E(\mathcal{M}, \tau)}$, and so $\varlimsup_{n}\left\||x|+\lambda u^{*} y_{n}\right\|_{E(\mathcal{M}, \tau)} \leq 1$. By Lemma 1.5, it follows that

$$
\lim _{n}\left\||x|+\lambda u^{*} y_{n}\right\|_{E(\mathcal{M}, \tau)}=1 .
$$


Similarly, using the polar decomposition $x^{*}=u^{*}\left|x^{*}\right|$ of $x^{*}$, one can show that

$$
\lim _{n}\left\|\left|x^{*}\right|+\lambda u y_{n}^{*}\right\|_{E(\mathcal{M}, \tau)}=1 .
$$

Since $\mu(|x|)=\mu\left(\left|x^{*}\right|\right)$ is a $\mathbb{C}$-LUR point, by Case 2 we conclude that

$$
\left\|u^{*} y_{n}\right\|_{E(\mathcal{M}, \tau)} \rightarrow 0 \quad \text { and } \quad\left\|u y_{n}^{*}\right\|_{E(\mathcal{M}, \tau)}=\left\|y_{n} u^{*}\right\|_{E(\mathcal{M}, \tau)} \rightarrow 0 .
$$

Hence, in view of $s(x)=u^{*} u$ and $s\left(x^{*}\right)=u u^{*}$ we have

$$
\left\|s\left(x^{*}\right) y_{n}\right\|_{E(\mathcal{M}, \tau)} \rightarrow 0 \quad \text { and } \quad\left\|y_{n} s(x)\right\|_{E(\mathcal{M}, \tau)} \rightarrow 0 .
$$

It is also easy to check that

$$
\left|x+\lambda n\left(x^{*}\right) y_{n} n(x)\right|=|||x|+\lambda\left|n\left(x^{*}\right) y n(x)\right||| .
$$

Combining the above and the equality $\mathbf{1}=n\left(x^{*}\right)+s\left(x^{*}\right)=n(x)+s(x)$, we get

$$
\begin{aligned}
\left\||x|+\lambda\left|n\left(x^{*}\right) y_{n} n(x)\right|\right\|_{E(\mathcal{M}, \tau)}= & \left\|x+\lambda n\left(x^{*}\right) y_{n} n(x)\right\|_{E(\mathcal{M}, \tau)} \\
\leq & \left\|x+\lambda y_{n}\right\|_{E(\mathcal{M}, \tau)}+\left\|s\left(x^{*}\right) y_{n} s(x)\right\|_{E(\mathcal{M}, \tau)} \\
& +\left\|s\left(x^{*}\right) y_{n} n(x)\right\|_{E(\mathcal{M}, \tau)} \\
& +\left\|n\left(x^{*}\right) y_{n} s(x)\right\|_{E(\mathcal{M}, \tau)} \rightarrow 1 .
\end{aligned}
$$

Again, Lemma 1.5 yields $\lim _{n}\left\||x|+\lambda\left|n\left(x^{*}\right) y_{n} n(x)\right|\right\|_{E(\mathcal{M}, \tau)}=1$, and by the first case, $\left\|n\left(x^{*}\right) y_{n} n(x)\right\|_{E(\mathcal{M}, \tau)} \rightarrow 0$. Hence

$$
\begin{aligned}
& \left\|y_{n}\right\|_{E(\mathcal{M}, \tau)} \\
& \quad \leq\left\|n\left(x^{*}\right) y_{n} n(x)\right\|_{E(\mathcal{M}, \tau)}+2\left\|s\left(x^{*}\right) y_{n}\right\|_{E(\mathcal{M}, \tau)}+\left\|y_{n} s(x)\right\|_{E(\mathcal{M}, \tau)} \rightarrow 0,
\end{aligned}
$$

and the proof is complete.

TheOREM 4.2. Suppose that the trace $\tau$ on $\mathcal{M}$ is $\sigma$-finite. If $x$ is a $\mathbb{C}$-LUR point of $B_{E(\mathcal{M}, \tau)}$ then $\mu(x)$ is a $\mathbb{C}-L U R$ point of $B_{E}$ and either

(i) $\mu_{\infty}(x)=0$, or

(ii) $n(x) \mathcal{M} n\left(x^{*}\right)=0$ and $|x| \geq \mu_{\infty}(x) s(x)$.

Proof. Since every $\mathbb{C}$-LUR point is a $\mathbb{C}$-extreme point, by Theorem 3.10 , (i) or (ii) is satisfied. The fact that $\mu(x)$ is a $\mathbb{C}$-LUR point whenever $x$ is $\mathbb{C}$-LUR can be proved analogously to the corresponding statement about strongly extreme points in Theorem 2.7, replacing $\lambda= \pm 1$ with $\lambda= \pm 1, \pm i$.

COROllary 4.3. Let $E$ be an order continuous symmetric space on $[0, \alpha), \alpha=\tau(\mathbf{1})$, and $\mathcal{M}$ be a von Neumann algebra with a faithful, normal, $\sigma$-finite trace $\tau$. Then $E$ is a $\mathbb{C}$ - $L U R$ space if and only if $E(\mathcal{M}, \tau)$ is a $\mathbb{C}$-LUR space.

REMARK 4.4. Note that if $E$ is order continuous then $E \subset E_{0}$, and the norm on $E$ is strongly symmetric. 
TheOREM 4.5. Let $E \subset c_{0}$ be an order continuous symmetric sequence space. Then $C_{E}$ is a $\mathbb{C}$-LUR space if and only if $E$ is a $\mathbb{C}$ - $L U R$ space.

Proof. Since $E$ is isometrically embedded in $C_{E}$, it follows that if $C_{E}$ is a $\mathbb{C}$-LUR space then so is $E$. Now $C_{E}=G(B(H), \operatorname{tr})$ and $\|x\|_{C_{E}}=$ $\|x\|_{G(B(H), \operatorname{tr})}$ for any compact operator $x$. Hence the proof of the converse implication is analogous to the proof of Theorem 4.1 .

5. $\mathbb{C}$-LUR and $\mathbb{C}$-MLUR properties. Let us discuss here the notions of complex strongly extreme points and complex midpoint locally uniformly rotund spaces. One can define a modulus of complex strong extremality analogously to the modulus of strong extremality in the real case, introduced by $\mathrm{C}$. Finet in [20]. Let $(X,\|\cdot\|)$ be a Banach space over the field of complex numbers. For $x \in S_{X}$ and $\epsilon>0$, the modulus of complex strong extremality at $x$ is the number

$$
\Delta(x, \epsilon)=\inf \{1-|\lambda|: \exists y,\|y\|>\epsilon,\|\lambda x \pm y\| \leq 1 \text { and }\|\lambda i x \pm y\| \leq 1\} .
$$

The element $x \in S_{X}$ is said to be a $\mathbb{C}-M L U R$ point of $B_{X}$, or a complex strongly extreme point of $B_{X}$, if $\Delta(x, \epsilon)>0$ for any $\epsilon>0$. A Banach space $X$ is said to be complex midpoint locally uniformly rotund, or a $\mathbb{C}-$ MLUR space, if every element of $S_{X}$ is a $\mathbb{C}$-MLUR point.

We will demonstrate that the notions of $\mathbb{C}$-LUR and $\mathbb{C}$-MLUR points, and hence the notions of $\mathbb{C}$-LUR and $\mathbb{C}$-MLUR spaces, are equivalent in any complex Banach space. Consequently, in complex Banach spaces these complex properties are not distinguishable, unlike the corresponding properties LUR and MLUR [29]. We need the following lemma.

Lemma 5.1. An element $x \in S_{X}$ is a $\mathbb{C}-M L U R$ point if and only for any $\left\{x_{n}\right\} \subset X,\left\|x+\lambda x_{n}\right\| \rightarrow 1$ for all $\lambda= \pm 1, \pm i$ implies that $\left\|x_{n}\right\| \rightarrow 0$.

Proof. Suppose that $x$ is a $\mathbb{C}$-MLUR point, that is, $\Delta(x, \epsilon)>0$ for all $\epsilon>0$. Let $\left\|x \pm x_{n}\right\| \rightarrow 1$ and $\left\|x \pm i x_{n}\right\| \rightarrow 1$, where $\left\{x_{n}\right\} \subset X$. Set

$$
c_{n}=\max _{\lambda \in\{ \pm 1, \pm i\}}\left\|x+\lambda x_{n}\right\| .
$$

Clearly, $c_{n} \rightarrow 1$. If $c_{n} \leq 1$ for some $n$, then $\left\|x+\lambda x_{n}\right\| \leq 1$ for all $\lambda= \pm 1, \pm i$, and consequently $x_{n}=0$. Indeed, suppose that $x_{n} \neq 0$. Then there exists an $\epsilon>0$ such that $\left\|x_{n}\right\|>\epsilon,\left\|x \pm x_{n}\right\| \leq 1$ and $\left\|i x \pm x_{n}\right\| \leq 1$. But then $\Delta(x, \epsilon)=0$, a contradiction. Therefore we can assume that $c_{n}>1$ for all $n \in \mathbb{N}$. Clearly, for all $n \in \mathbb{N}$,

$$
\left\|c_{n}^{-1} x \pm c_{n}^{-1} x_{n}\right\| \leq 1 \quad \text { and } \quad\left\|i c_{n}^{-1} x \pm c_{n}^{-1} x_{n}\right\| \leq 1 .
$$

Denote $\lambda_{n}=c_{n}^{-1}, n \in \mathbb{N}$. Then for each $n$, the element $a_{n}=c_{n}^{-1} x_{n}$ satisfies $\left\|\lambda_{n} x \pm a_{n}\right\| \leq 1$ and $\left\|i \lambda_{n} x \pm a_{n}\right\| \leq 1$. Hence $\left\|a_{n}\right\| \rightarrow 0$ and consequently $\left\|x_{n}\right\| \rightarrow 0$ : if not, then there exists an $\epsilon>0$ and a subsequence $a_{n_{k}}$ such 
that $\left\|a_{n_{k}}\right\|>\epsilon$, and since $\lambda_{n} \rightarrow 1$,

$$
\Delta(x, \epsilon)=\inf \{1-|\lambda|: \exists y,\|y\|>\epsilon,\|\lambda x \pm y\| \leq 1 \text { and }\|\lambda i x \pm y\| \leq 1\}=0 .
$$

To prove the converse implication, assume that $\Delta(x, \epsilon)=0$ for some $\epsilon>0$. Then there exists a sequence $\left\{\lambda_{n}\right\} \subset \mathbb{C}$ satisfying $\left|\lambda_{n}\right| \uparrow 1$ and for all $n \in \mathbb{N}$, there is an $x_{n} \in B_{X}$ with $\left\|x_{n}\right\| \geq \epsilon$ such that $\left\|\lambda_{n} x \pm x_{n}\right\| \leq 1$ and $\left\|i \lambda_{n} x \pm x_{n}\right\| \leq 1$. Therefore, for all $n \in \mathbb{N}$ we have

$$
\left\|x \pm \lambda_{n}^{-1} x_{n}\right\| \leq\left|\lambda_{n}\right|^{-1} \text { and }\left\|x \pm i \lambda_{n}^{-1} x_{n}\right\| \leq\left|\lambda_{n}\right|^{-1}
$$

and since $\left|\lambda_{n}\right| \rightarrow 1, \varlimsup_{n}\left\|x \pm \lambda_{n}^{-1} x_{n}\right\| \leq 1$ and $\overline{\lim }_{n}\left\|x \pm i \lambda_{n}^{-1} x_{n}\right\| \leq 1$. As $\|x\|=1$, Lemma 1.5 yields $\lim _{n}\left\|x \pm \lambda_{n}^{-1} x_{n}\right\|=1$ and $\lim _{n}\left\|x \pm i \lambda_{n}^{-1} x_{n}\right\|=1$. Hence there exists a subsequence $\left\{\lambda_{n_{k}}^{-1} x_{n_{k}}\right\}$ with $\lim _{k}\left\|\lambda_{n_{k}}^{-1} x_{n_{k}}\right\| \neq 0$ such that $\lim _{k}\left\|x \pm \lambda_{n_{k}}^{-1} x_{n_{k}}\right\|=1$ and $\lim _{k}\left\|x \pm i \lambda_{n_{k}}^{-1} x_{n_{k}}\right\|=1$.

Now we can state the equivalence of $\mathbb{C}$-LUR and $\mathbb{C}$-MLUR properties.

Proposition 5.2. Let $x \in S_{X}$. The following conditions are equivalent:

(i) $x$ is a $\mathbb{C}$ - $L U R$ point of $B_{X}$.

(ii) For all $\epsilon>0$ there exists $\delta(\epsilon)>0$ such that for all $y \in X$,

$$
\sup _{\lambda= \pm 1, \pm i}\|x+\lambda y\|<1+\delta \quad \text { implies }\|y\|<\epsilon .
$$

(iii) For all $\left\{y_{n}\right\} \subset X, \sup _{\lambda= \pm 1, \pm i}\left\|x+\lambda y_{n}\right\| \rightarrow 1$ implies $\left\|y_{n}\right\| \rightarrow 0$.

(iv) For all $\left\{y_{n}\right\} \subset X,\left\|x \pm y_{n}\right\| \rightarrow 1$ and $\left\|x \pm i y_{n}\right\| \rightarrow 1$ implies $\left\|y_{n}\right\| \rightarrow 0$.

(v) $x$ is a $\mathbb{C}$-MLUR point of $B_{X}$.

Proof. It is clear that (ii) implies (i), (iii) implies (iv) and conditions (ii) and (iii) are equivalent. By Lemma 5.1, conditions (iv) and (v) are also equivalent. It remains to show (i) $\Rightarrow$ (ii) and (iv) $\Rightarrow$ (iii).

Let $\epsilon>0$ and suppose that (i) holds. Hence for $\epsilon / 2>0$, there exists $\delta(\epsilon)>0$ such that for any $y \in X, \sup _{|\lambda| \leq 1}\|x+\lambda y\|<1+\delta(\epsilon)$ implies that $\|y\|<\epsilon / 2$. Assume now that $\sup _{\lambda= \pm 1, \pm i}\|x+\lambda y\|<1+\delta$. Then for $-1 \leq c \leq 1$ and $\lambda= \pm 1, \pm i$ we have

$$
\|x+c \lambda y\| \leq((1+c) / 2)\|x+\lambda y\|+((1-c) / 2)\|x-\lambda y\|<1+\delta .
$$

Hence for all $c \in \mathbb{R}$ with $|c| \leq 1,\|x+c y\|<1+\delta$ and $\| x+$ ciy $\|<1+\delta$. Let $\lambda=a+b i \in \mathbb{C}$, with $|\lambda| \leq 1$. Since $|a|,|b| \leq 1$ it follows that

$$
\|x+\lambda y / 2\|=\| x+a y / 2+\text { biy } / 2\|\leq\| x+\text { ay }\|/ 2+\| x+\text { biy } \| / 2<1+\delta .
$$

Consequently, $\sup _{|\lambda|<1}\|x+y / 2\|<1+\delta$ and by (i), $\|y / 2\|<\epsilon / 2$ and so $\|y\|<\epsilon$. Therefore (ii) is satisfied.

To show (iv) $\Rightarrow$ (iii), suppose that $\left\{y_{n}\right\} \subset X$ and $\sup _{\lambda= \pm 1, \pm i}\left\|x+\lambda y_{n}\right\|$ $\rightarrow 1$. Then $\varlimsup_{n}\left\|x \pm y_{n}\right\| \leq 1$ and $\overline{\lim }_{n}\left\|x \pm i y_{n}\right\| \leq 1$. Thus by Lemma 1.5 . for all $\lambda= \pm 1, \pm i$ we have $\lim _{n}\left\|x+\lambda y_{n}\right\|=1$, and so by (iv), $\left\|y_{n}\right\| \rightarrow 0$. 
We finish with examples showing that complex uniform rotundity, complex local uniform rotundity and complex rotundity do not coincide.

EXAmple 5.3. (1) The space $E=\left(\ell_{\infty},\|\cdot\|\right)$ equipped with the norm $\|x\|=\|x\|_{\infty}+\sum_{n=1}^{\infty}|x(n)| / 2^{n-1}$ is complex rotund but not complex locally uniformly rotund. One can show easily [26] that the unit vector $2^{-1} e_{1}=$ $\left(2^{-1}, 0, \ldots\right)$ is an UM point in $B_{E_{r}}$, so it is $\mathbb{C}$-extreme point in $B_{E}$, but not an ULUM point so not a $\mathbb{C}$-LUR point (Theorem 1.4).

(2) Orlicz-Lorentz spaces $\Lambda_{\varphi, w}$ are locally uniformly rotund and hence $\mathbb{C}$-LUR whenever $\varphi$ is strictly convex and satisfies condition $\Delta_{2}$ [4]. However if they are complex uniformly rotund then in addition to those conditions on $\varphi$, the weight $w$ must be regular [8]. So there exist Orlicz-Lorentz spaces that are $\mathbb{C}$-LUR but not complex uniformly rotund.

Acknowledgments. We wish to express our gratitude to Peter Dodds, Ben de Pagter and Fedor Sukochev, who allowed us to use their unpublished manuscript of the monograph Theory of Noncommutative Integration [18]. It was of tremendous help to learn noncommutative theory of measurable operators from this well ordered material. Our special thanks go to Fedor Sukochev who was a wonderful host in Spring 2010, while we were visiting the University of New South Wales in Sydney. We thank him also for his valuable comments concerning this paper that improved it considerably.

\section{References}

[1] M. D. Acosta, R. Aron and L. A. Moraes, Boundaries for spaces of holomorphic functions on $M$-ideals in their biduals, Indiana Univ. Math. J. 58 (2009), 2575-2595.

[2] J. Arazy, On the geometry of the unit ball of unitary matrix spaces, Integral Equations Operator Theory 4 (1981), 151-171.

[3] C. Bennett and R. Sharpley, Interpolation of Operators, Pure Appl. Math. 129, Academic Press, Boston, MA, 1988.

[4] J. Cerda, H. Hudzik, A. Kamińska and M. Mastyło, Geometric properties of symmetric spaces with applications to Orlicz-Lorentz spaces, Positivity 2 (1998), 311-337.

[5] V. I. Chilin, A. V. Krygin, and F. A. Sukochev, Extreme points of convex fully symmetric sets of measurable operators, Integral Equations Operator Theory 15 (1992), 186-226.

[6] - - - - , Local uniform and uniform convexity of noncommutative symmetric spaces of measurable operators, Math. Proc. Cambridge Philos. Soc. 111 (1992), $355-368$.

[7] V. I. Chilin and F. A. Sukochev, Weak convergence in non-commutative symmetric spaces, J. Operator Theory 31 (1994), 35-65.

[8] C. Choi, A. Kamińska and H. J. Lee, Complex convexity of Orlicz-Lorentz spaces and its applications, Bull. Polish Acad. Sci. Math. 52 (2004), 19-38.

[9] W. J. Davis, D. J. H. Garling and N. Tomczak-Jaegermann, The complex convexity of quasi-normed linear spaces, J. Funct. Anal. 55 (1984), 110-150. 
[10] S. J. Dilworth, Complex convexity and the geometry of Banach spaces, Math. Proc. Cambridge Philos. Soc. 99 (1986), 495-506.

[11] P. G. Dodds and T. K. Dodds, Some aspects of the theory of symmetric operator spaces, in: First International Conference in Abstract Algebra (Kruger Park, 1993), Quaestiones Math. 18 (1995), 47-89.

[12] P. G. Dodds, T. K. Dodds, and B. de Pagter, Noncommutative Banach function spaces, Math. Z. 201 (1989), 583-597.

[13] - - - - Fully symmetric operator spaces, Integral Equations Operator Theory 15 (1992), 942-972.

[14] —, - - , Noncommutative Köthe duality, Trans. Amer. Math. Soc. 339 (1993), $717-750$.

[15] P. G. Dodds, T. K. Dodds, P. N. Dowling, C. J. Lennard and F. A. Sukochev, A uniform Kadec-Klee property for symmetric operator spaces, Math. Proc. Cambridge Philos. Soc. 118 (1995), 487-502.

[16] P. G. Dodds, T. K. Dodds and F. A. Sukochev, Lifting of Kadec-Klee properties to symmetric spaces of measurable operators, Proc. Amer. Math. Soc. 125 (1997), 1457-1467.

[17] -, - - - Banach-Saks properties in symmetric spaces of measurable operators, Studia Math. 178 (2007), 125-166.

[18] P. G. Dodds, B. de Pagter and F. A. Sukochev, Theory of Noncommutative Integration, unpublished monograph, to appear.

[19] T. Fack and H. Kosaki, Generalized s-numbers of $\tau$-measurable operators, Pacific J. Math. 123 (1986), 269-300.

[20] C. Finet, Uniform convexity properties of norms on a super-reflexive Banach space, Israel J. Math. 53 (1986), 81-92.

[21] J. Globevnik, On complex strict and uniform convexity, Proc. Amer. Math. Soc. 47 (1975), 175-178.

[22] I. C. Gohberg and M. G. Krein, Introduction to the Theory of Linear Nonselfadjoint Operators, Amer. Math. Soc., Providence, RI, 1969.

[23] H. Hudzik and A. Narloch, Relationships between monotonicity and complex rotundity properties with some consequences, Math. Scand. 96 (2005), 289-306.

[24] R. V. Kadison and J. R. Ringrose, Fundamentals of the Theory of Operator Algebras. Vol. I, Grad. Stud. in Math. 15, Amer. Math. Soc., Providence, RI, 1997.

[25] N. J. Kalton and F. A. Sukochev, Symmetric norms and spaces of operators, J. Reine Angew. Math. 621 (2008), 81-121.

[26] P. Kolwicz and R. Płuciennik, Points of upper local uniform monotonicity in Calderón-Lozanovskii spaces, J. Convex Anal. 17 (2010), 111-130.

[27] S. G. Kreı̆n, Yu. İ. Petunīn, and E. M. Semënov, Interpolation of Linear Operators, Transl. Math. Monogr. 54, Amer. Math. Soc., Providence, RI, 1982.

[28] H. J. Lee, Monotonicity and complex convexity in Banach lattices, J. Math. Anal. Appl. 307 (2005), 86-101.

[29] P. K. Lin, Köthe-Bochner Function Spaces, Birkhäuser Boston, Boston, MA, 2004.

[30] B. de Pagter, Non-commutative Banach function spaces, in: Positivity, Trends Math., Birkhäuser, Basel, 2007, 197-227.

[31] - Notes on traces in tensor products, private communication, to appear.

[32] W. F. Stinespring, Integration theorems for gages and duality for uni-modular groups, Trans. Amer. Math. Soc. 90 (1959), 15-56.

[33] F. A. Sukochev and V. I. Chilin, The triangle inequality for operators that are measurable with respect to Hardy-Littlewood order, Izv. Akad. Nauk UzSSR Ser. Fiz.-Mat. Nauk 1988, no. 4, 44-50. 
[34] M. Takesaki, Theory of Operator Algebras. I, Springer, New York, 1979.

[35] E. Thorp and R. Whitley, The strong maximum modulus theorem for analytic functions into a Banach space, Proc. Amer. Math. Soc. 18 (1967), 640-646.

[36] N. Tomczak-Jaegermann, Uniform convexity of unitary ideals, Israel J. Math. 48 (1984), 249-254.

[37] T. Wang and Y. Teng, Complex locally uniform rotundity of Musielak-Orlicz spaces, Sci. China Ser. A 43 (2000), 113-121.

[38] Q. H. Xu, Analytic functions with values in lattices and symmetric spaces of measurable operators, Math. Proc. Cambridge Philos. Soc. 109 (1991), 541-563.

Małgorzata Marta Czerwińska, Anna Kamińska

Department of Mathematical Sciences

The University of Memphis

Memphis, TN 38152, U.S.A.

E-mail: mmczrwns@memphis.edu

kaminska@memphis.edu

Received July 9, 2010

Revised version October 21, 2010 